\title{
FRACTURE PROBLEMS IN COMPOSITE MATERIALS
}

\author{
BY \\ F. ERDOGAN
}

TECHNicAL REPORT NASA-TR-72-2

ApRIL 1972
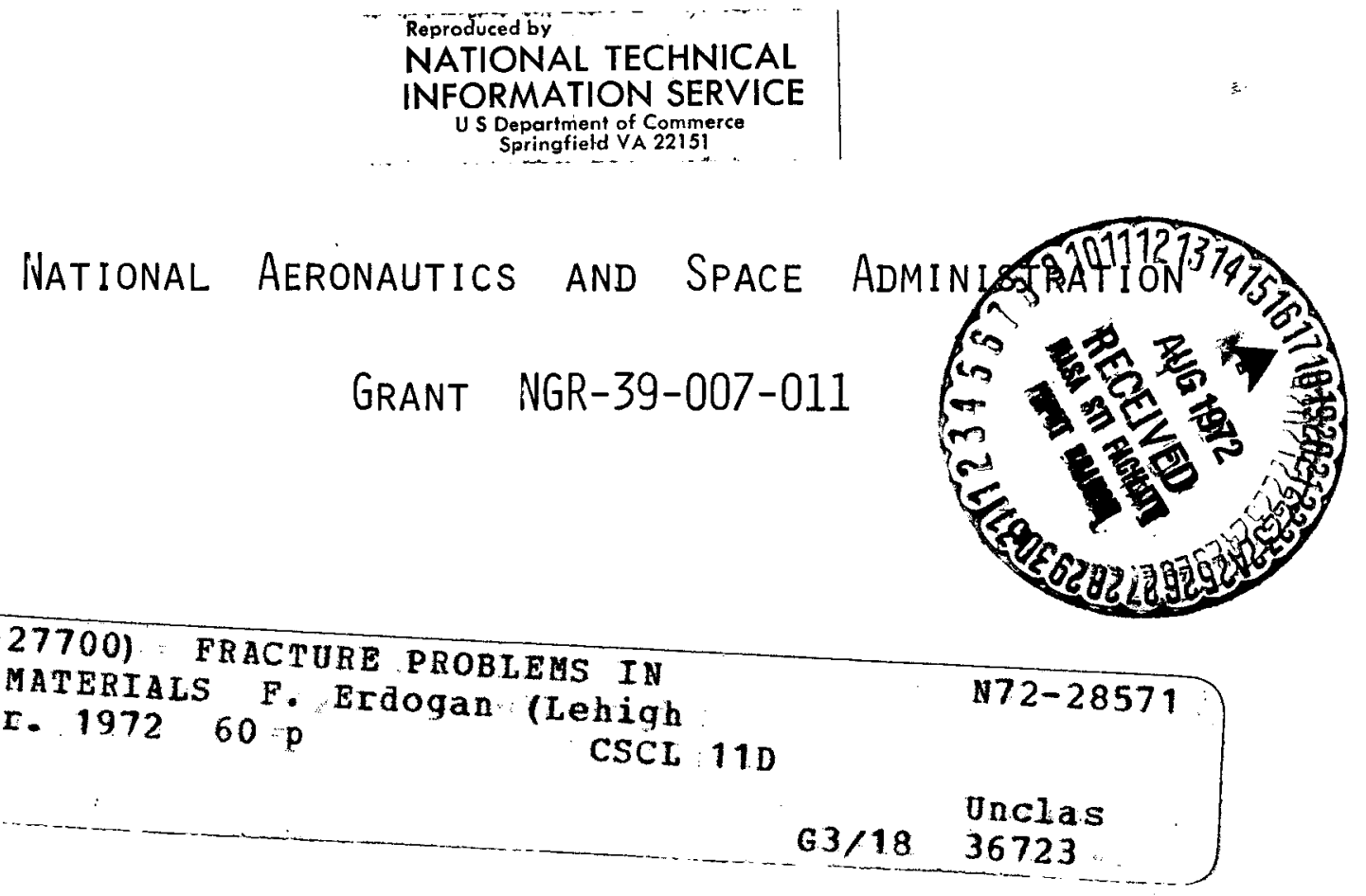


\section{FRACTURE PROBLEMS IN COMPOSITE MATERIALS*}

by F. Erdogan

Lehigh University

Bethlehem, Penna.

\section{ABSTRACT}

In this paper a series of fracture problems in composite materials are identified, their methods of solution are briefly discussed, and some sample results are presented. The main problem of interest is the determination of the stress state in the neighborhood of localized imperfections such as cracks and inclusions which may exist in the composite. Particular emphasis is placed on the evaluation of quantities such as the stress intensity factors, the power of the stress singularity, and the strain energy release rate, which may be used directly or indirectly in connection with an appropriate fracture criterion for the prediction of fracture initiation and propagation load levels. The topics discussed in the paper include a crack in layered composites, a crack terminating at and going through a bi-material interface, a penny-shaped crack in a filamentreinforced elastic matrix, and inclusion problems in bonded materials.

* The research reported in this work was supported by The National Science Foundation under Grant GK-11977 and by the National Aeronautics and Space Administration under Grant NGR-39-007-011. 


\section{INTRODUCTION}

Generally the fracture of composite materials may be considered from two different points of view. In the first approach the main interest is in estimating or studying the "bulk strength" of a given structure or of a part under a known system of external loads and environmental conditions. In this type of studies it is usually assumed that the material is statistically homogeneous and existing imperfections (which are unavoidable) are randomly distributed. Here, the very nature of the problem requires that some kind of statistical strength theory be used as a guide in the investigations.

In the second approach to the fracture studies in composites, one is basically interested in the initiation of fracture propagation from the "localized" imperfections which are known (or assumed) to exist in the material. Here, before embarking on any elaborate analysis, for this local fracture initiation one has to adopt a proper fracture criterion and decide on the type of "load factor" to be evaluated. Usually the fracture criterion consists of a simple comparison between a calculated load factor and a material constant which is determined from certain standard experiments. Hence, the approach is, of necessity, deterministic.

In this paper we are interested only in the second type of problems, namely, the fracture problems arising from isolated imperfections. The most common forms of these imperfections. which may be found in composites are voids, cracks, and inclusions with varying degrees of relative stiffness. In studying 
the composites which consist of perfectly bonded multiphase elastic continua, it is usually assumed that the imperfection located near to or on an interface may be idealized as a crack or as a flat inclusion, the reason being partly analytical. As in most analytical work on the subject, in this paper we will restrict our attention to the linearly elastic materials and concentrate our numerical effort on the evaluation of the strength of the stress singularities known as the stress intensity factors." These factors are defined in terms of (or, are obtained from) the asymptotic expressions for the stress state around the singular points, which are generally of the following form:

$$
\sigma_{i j}(r, \theta)=\frac{k_{1}}{(2 r)^{\alpha}} f_{i j}^{1}(r, \theta)+\frac{k_{2}}{(2 r)^{\alpha}} f_{i j}^{2}(r, \theta)+0\left(r^{1-\alpha}\right)
$$

where $k_{1}$ and $k_{2}$ are the stress intensity factors, $(r, \theta)$ are the polar coordinates in the plane perpendicular to the periphery of the crack or of the inclusion, $f_{i j} k,(k=1,2)$ are bounded functions, and $\alpha$ is the power of the singularity $(0<\alpha<1)$. The conjecture here is that, regardless of the nature of the singularity at the imperfection front, in at least ideally brittle materials $k_{1}$ and $k_{2}$ are a reasonable measure of the severity of deformations and stresses at the expected location of the fracture initiation, and hence should provide sufficient information for the calculation of the "load factor" which may be used in a proper fracture criterion.

Figure 1 shows various possible modes of crack initiation in the neighborhood of a singular point. Figure la describes the 
most probabie crack propagation mode for a crack imbedded in a symmetrically loaded homogeneous. (and from the viewpoint of fracture resistance, isotropic) medium. Here, as the crack propagates, the stress state around the crack tip remains autonomous or self-similar, the only change being in the stress intensity factor. Therefore, in this case, if one prefers to use it, an energy balance type of fracture criterion is applicable. The only other fracture mode for which this could be said is the propagation of an interface crack shown in Figure $1 c$. Here, $\left(k_{1}{ }^{2}+k_{2}^{2}\right)$ is the measure of the strain energy release rate and may be considered as an appropriate load factor [1]. The type of fracture shown in Figure $1 \mathrm{~b}$ may initiate from a crack tip around which the stress state is not symmetric. In bonded materials containing an interface flaw, if the adhesive strength of the bond is greater than the rupture strength of one of the adjacent materials, the fracture mode may be that described by Figure $1 d$. Figure 1 e shows the fracture initiation around the end point of a flat elastic inclusion. The common feature of the stress singularities in fracture problems described by Figures la-le is that the power of the singularity a shown in (1) is $1 / 2$.

On the other hand in the crack initiation problems for the flaws running into and that going through a bi-material interface shown in Figures $1 \mathrm{f}-1 \mathrm{i}$ this no longer is the case, i.e., if $\mu_{1} \neq \mu_{2}$ then $\alpha \neq 1 / 2$ where $\mu_{1}$ and $\mu_{2}$ are the shear moduli $\left(1>\alpha>1 / 2\right.$ for $\mu_{1}>\mu_{2}$ and $1 / 2>\alpha>0$ for $\mu_{1}<\mu_{2}$ in Figures $1 \mathrm{f}-1 \mathrm{~g}$, and $1 / 2>\alpha \geq 0$ for Figure $1 \mathrm{i})$. In the problem of the flaw running into an interface, whether further fracture 
propagation would be the cleavage of the adjacent medium ( $f$ ), debonding along the interface $(g)$, or reflection back into the first medium ( $h$ ) may again depend on the relative strength-toToad factor ratios for various possible fracture modes. The same principle also applies to the initiation of debonding crack shown in $(i)$.

In this paper we will give the results regarding $\alpha, k_{n}$, and $f_{i j},(i, j, n=1,2)$ for various problems arising from the fracture of composite materials. As to how to use these results in practice, it may depend on one's preference for a particular fracture criterion. However, a certain comparative test common to all fracture modes shown in Figure 1 is worth noting. Whatever the nature of the conjectured fracture mechanism (namely, cleavage starting from the singular point, or fracture initiation and propagation through hole or craze formation and coalescence around the singular point), the intensity of the (tensile) stress field within the immediate neighborhood of the singular point will be the major factor controlling the fracture initiation, and this stress field intensity is clearly dependent on $k_{i} / r^{\alpha},(i=1,2)$ (see equation (1)). Hence, comparing, for example, the maximum value of the cleavage stress $\sigma_{\theta \theta}$ (in the homogeneous medium) or an interface stress vector computed in terms of $k_{i}$ and $\alpha$ at a characteristic distance from the singular point, with the corresponding quantities obtained from idealized test configurations under rupture conditions may provide a rather simple criterion for fracture initiation. 


\section{GENERAL METHOD OF SOLUTION}

Assuming that the solution to the elastostatic problem for the imperfection-free nonhomogeneous solid is available, the singular behavior of the solution around an isolated imperfection may be obtained from the stress disturbance or perturbation prob1em. If desired, the complete solution may then be found through superposition. The perturbation problem is a mixed boundary value problem and can always be formulated in terms of a system of singular integral equations. In the case of plane strain, the generalized plane stress, and the axisymmetric problems for the type of imperfections shown in Figure 1 (and for many other problems), the general form of these integral equations may be expressed as follows:

$$
\begin{aligned}
& \sum_{1}^{2} a_{i j} \phi_{j}(x)+\frac{1}{\pi} \int_{-a}^{a} \sum_{1}^{2} b_{i j} \phi_{j}(t) \frac{d t}{t-x} \\
& +\frac{1}{\pi} \int_{-a}^{a} \sum_{j=1}^{2} \sum_{k=1}^{3}\left[c_{i j k} \phi_{j}(t) \frac{(x+a)^{k-1}}{(t+x+2 a)^{k}}+d_{i j k} \phi_{j}(t) \frac{(a-x)^{k-1}}{(t+x-2 a)^{k}}\right] d t \\
& +\int_{-a}^{a} k_{i j}(x, t) \phi_{j}(t) d t=\gamma_{i} f_{j}(x), \quad(i=1,2),-a<x<1
\end{aligned}
$$

where $a$ is the half-length or the radius of the imperfection and $a_{i j}, b_{i j}, c_{i j k}, d_{i j k}$, and $\gamma_{i}(i, j=1,2, k=1,2,3)$ are constants which are dependent on the elastic properties of the materials forming the composite. In crack problems the input functions $f_{i}$, $(i=1,2)$ are the components of the traction vector on the crack 
surface and the unknown functions $\dot{\phi}_{j}$ are defined by [2-8]

$$
\phi_{i}(x)=\frac{\partial}{\partial x}\left(u_{i}^{+}-u_{i}^{-}\right), \quad(i=1,2)
$$

where $u_{1}$ and $u_{2}$ are the crack surface displacements parallel and perpendicular to the plane of the crack, respectively, $x$ is the (rectangular or radius) coordinate in the plane of the crack, and superscripts + and - refer to the opposite faces of the crack. Note that in this case $\phi_{j}(i=1,2)$ may also be interpreted as dislocation densities.

In the inclusion problems the input functions and the unknown functions are generally defined by [9]

$$
\begin{array}{ll}
f_{k}(x)=-\frac{\partial}{\partial x} u_{m k}, & (k=1,2), \\
\phi_{1}(x)=\sigma_{12}{ }^{+}-\sigma_{12} & , \quad \phi_{2}(x)=\sigma_{22}{ }^{+}-\sigma_{22}{ }^{-},
\end{array}
$$

where $u_{m 1}$ and $u_{m 2}$ are the components, of the displacement vector in the inclusion-free matrix at the location of the inclusion obtained from the given system of external loads, and $\sigma_{12}, \sigma_{22}$ are, respectively, the shear and the normal stress acting on the surface of the inclusion, the superscripts + and - again referring to the opposite faces of the inclusion.

In (2) the kernels $k_{i j},(i, j=1,2)$ are known functions which are bounded in plane problems and have logarithmic singularities in axisymmetric problems. In either case they are treated as simple Fredholm kernels. 'If the crack or the inclusion is completely imbedded in: a homogeneous medium, then the constants 
$a_{i j}(i, j=1,2)$ vanish and the kernels in the third integral in (2) are bounded. In this case the system of integral equations has simple Cauchy kernels and the solution has $r^{-1 / 2}$ type singularity $[2,3,5,7]$. If the imperfection lies along the interface, then the constants $c_{i j k}$ and $d_{i j k}$ are zero, the system of singular integral equations are of the second kind and the solution has the following typical oscillating singularity with a power $-1 / 2$ :

$$
\left(\phi_{k}, \sigma_{i j}\right) \sim r^{-1 / 2} \cos [\omega \sin [\log (r / 2 a)] .
$$

If an imperfection end terminates at an interface, from (2) it is seen that the kernels in the third integral become unbounded (as $x^{-1}$ ) as $x$ and $t$ approach the end point simultaneously. In this case the system of integral equations is said to have generalized Cauchy kernels, the power of the singularity of the solution is no longer $-1 / 2$, and the solution of the system is not straightforward and requires much more care [7].

In all the problems discussed here, it is not difficult to show that the index of the system of singular integral equations (2) is +1 , hence the solution of the problem will contain two arbitrary constants [10]. These constants are determined by using the conditions

$$
\int_{-a}^{a} \phi_{k}(x) d x=0, \quad(k=1,2) .
$$

In crack problems (7) expresses the conditions of single-valuedness of displacements, and in inclusion problems it constitutes 
the conditions of static equilibrium of the inclusion.

For the system of singular integral equations with simple Cauchy kernels the direct regularization technique in the type of problems under consideration becomes extremely cumbersome, and for the equations with generalized Cauchy kernels none exists. In practice these equations may be solved numerically in a very straightforward manner by using the methods described in [11-13]. In these techniques the function-theoretic method as described in [10] is applied directly to the integral equations and the fundamental functions of the system are obtained. Noting that these functions are the weights of certain Jacobi polynomials, and using the properties of the Jacobi polynomials, a set of Gauss-Jacobi type integration formulas are developed for the singular integrals and the system is solved numerically. Since the fundamental functions contain all the information regarding the singular behavior of the solution, and since they are determined exactly, the resulting numerical solution preserves the correct nature of the singularity.

\section{CRACK PROBLEMS IN LAYERED COMPOSITES}

In plane or axisymmetric problems for layered composites containing a crack completely imbedded into a homogeneous medium and lying parallel to the interfaces, the fundamental functions and the solution of the system of singular integral equations are of the following form 


$$
\begin{aligned}
& w_{1}(x)=w_{2}(x)=w(x)=\left(a^{2}-x^{2}\right)^{-1 / 2}, \\
& \phi_{k}(x)=w(x) F_{k}(x), \quad(k=1,2) .
\end{aligned}
$$

where the bounded functions $F_{k}(x),(k=1,2)$ are determined from the solution of the integral equations. In this problem the increased number of layers simply affects the computation of the Fredholm kernels $k_{i j}(x, t)$, otherwise presents no conceptual difficulty. The stress intensity factors defined by

$$
\begin{aligned}
& k_{1}(a)=\lim _{x \rightarrow a}[2(x-a)]^{1 / 2} \sigma_{y y}(x, 0), \\
& k_{2}(a)=\lim _{x \rightarrow a}[2(x-a)]^{1 / 2} \sigma_{x y}(x, 0),
\end{aligned}
$$

are easily shown to be related to the asymptotic values of $\phi_{1}$ and $\phi_{2}$ as follows:

$$
\begin{aligned}
& k_{1}(a)=-\frac{2 \mu}{1+k} \lim _{x \rightarrow a}[2(a-x)]^{1 / 2} \phi_{2}(x), \\
& k_{2}(a)=-\frac{2 \mu}{1+k} \lim _{x \rightarrow a}[2(a-x)]^{1 / 2} \phi_{1}(x),
\end{aligned}
$$

where $\mu$ is the shear modulus, $k=3-4 \nu$ for plane strain, and $\kappa=(3-\nu) /(1+\nu)$ for generalized plane stress, $\nu$ being the Poisson's ratio of the medium surrounding the crack.

In this problem the strain energy release rate for the crack propagating in its own plane may be expressed as

$$
\frac{\partial U}{\partial a}=\frac{\pi(1+k)}{4 \mu}\left(k_{1}^{2}+k_{2}^{2}\right) .
$$

From the viewpoint of fracture propagation in brittle materials, 
another important quantity is the "cleavage stress" $\sigma_{\theta \theta}$ given by [14]

$$
\sigma_{\theta \theta}(r, \theta)=\frac{1}{(2 r)^{1 / 2}} \cos \frac{\theta}{2}\left[k_{1} \cos ^{2} \frac{\theta}{2}-\frac{3}{2} k_{2} \sin \theta\right]+0\left(r^{1 / 2}\right)
$$

where $(r, \theta)$ are the polar coordinates at the crack tip.

If the crack is on the interface, the system of singular integral equations is of the second kind and the fundamental function is given by [3]

$$
\begin{aligned}
& w(x)=(a-x)^{\alpha}(a+x)^{\beta}, \\
& \alpha=-\frac{1}{2}-i \omega, \quad \beta=-\frac{1}{2}+i \omega, \quad \omega=\frac{1}{2 \pi} \log \left(\frac{1+\gamma}{1-\gamma}\right), \\
& \gamma=\frac{\left(\mu_{-}+\kappa_{-} \mu_{+}\right)-\left(\mu_{+}+\kappa_{+} \mu_{-}\right)}{\left(\mu_{-}+\kappa_{-} \mu_{+}\right)+\left(\mu_{+}+\kappa_{+} \mu_{-}\right)},
\end{aligned}
$$

where the subscripts + and - in the elastic constants refer to the materials on the $y>0$ and $y<0$ side of the crack, respectively. In this case, defining the stress intensity factors $k_{1}$ and $k_{2}$ by

$$
k_{1}+i k_{2}=\lim _{x \rightarrow a}[2(x-a)]^{1 / 2}\left(\frac{x-a}{x+a}\right)^{i \omega}\left[\sigma_{y y}(x, 0)+i \sigma_{x y}(x, 0)\right],
$$

the strain energy release rate for a crack propagating along the interface may be expressed as [1]

$$
\begin{aligned}
& \frac{\partial U}{\partial a}=\frac{\pi}{2} \frac{1+\kappa_{-}}{c \mu_{-}}\left(k_{1}^{2}+k_{2}^{2}\right), \\
& c=\frac{\mu_{+}-\mu_{-}}{\mu_{-}+\kappa_{-} \mu_{+}}+2-\frac{\kappa_{+} \mu_{-}-\kappa_{-} \mu_{+}}{\mu_{+}+\kappa_{+} \mu_{-}} .
\end{aligned}
$$


The functions $f_{i j}(r, \theta)$ shown in (1) which give the angular variation of the stresses around the crack tip may be found, for example, in [14] for the homogeneous materials and in [15] for an interface crack.

Figures 2-9 show some numerical results for various ( $p l a n e$ strain) configurations involving the materials steel ( $E=3 \times 10^{7}$ psi, $\nu=0.3)$, aluminum $\left(E=10^{7}\right.$ psi, $\left.\nu=0.3\right)$ and epoxy ( $E=$ $4.5 \times 10^{5}$ psi, $\left.\nu=0.35\right)$. The results are obtained for the perturbation problem in which the crack surface tractions are assumed to be

$$
\sigma_{y y}(x, 0)=-\sigma_{0}, \quad \sigma_{x y}(x, 0)=0 .
$$

Figure 2 shows that for the two (similar) half planes bonded through an epoxy layer which contains a symmetrically located crack, the stress intensity factors decrease with decreasing layer thickness. The opposite result is observed for the free layer, $k_{1}$ and $k_{2}$ going to infinity as $h \rightarrow 0$. The limiting value of the stress intensity factors, $\mu_{1}\left(1-v_{2}\right) /\left(1-v_{1}\right) \mu_{2}$, for $h \rightarrow 0$ is obtained by observing that the limit of the crack opening displacements in the layer 3 as $h \rightarrow 0$ is equal to that in the homogeneous $p l a n e 2$. If the crack is not in the mid-plane of the layer, $k_{2}$ is not zero. For a fixed ratio of $(h / 2 a)=1$, the results are shown in Figure 3 . In this and in the subsequent figures $\theta_{0}$ is the probable cleavage angle obtained from [16]

$$
\frac{\partial}{\partial \theta}\left[(2 r)^{1 / 2} \sigma_{\theta \theta}(r, \theta)\right]=0,
$$

where the cleavage stress $\sigma_{\theta \theta}$ is given by (13). The figure 
clearly shows that, for this symmetric geometry, the propagating crack would tend to stay in the mid-plane of the layer. The figure also shows a measure of the strain energy release rate $w_{3}$ defined by

$$
w_{3}=\frac{4 \mu_{3}}{a \pi \sigma_{0}^{2}\left(1+k_{3}\right)}\left(\frac{\partial U}{\partial a}\right)_{3}=\frac{k_{1}^{2}+k_{2}^{2}}{a \sigma_{0}^{2}} .
$$

As $h_{1} / h$ goes to 0 or 1 , the crack becomes an interface crack and the nature of the singularity changes. Thus at $h_{1}=0$ and at $h_{1}=h, k_{1}$ and $k_{2}$ (considered as functions of $h_{1}$ ) are not continuous. The quantity which is continuous is the strain energy release rate. Similar to (19) defining the measure of the, strain energy release rate for an interface crack (from (16)) by

$$
w_{23}=\frac{\left(k_{1}{ }^{2}+k_{2}{ }^{2}\right) 23}{\sigma_{0}{ }^{2} a}=\frac{2 c_{23} \mu_{3}}{a \pi \sigma_{0}{ }^{2}\left(1+k_{3}\right)}\left(\frac{\partial U}{\partial a}\right)_{23},
$$

the limiting values of $W_{3}$ may easily be found as follows:

$$
\lim _{h_{1} \rightarrow h} w_{3}=\frac{2}{c_{23}} w_{23}, \quad \lim _{h_{1} \rightarrow 0} w_{3}=\frac{2}{c_{13}} w_{13},
$$

where the constants $c_{23}$ and $c_{13}$ are given by (16.b) and $W_{23}$ and $W_{13}$ are obtained from the solution of the interface crack problem [4].

Results similar to that shown in Figures 2 and 3 are given in Figures 4 and 5 for three different materials. In this case too it is seen that (if $\mu_{3}<\mu_{1}, \mu_{3}<\mu_{2}$ ), for a given crack length as the layer thickness $h$ decreases the resistance of the 
bonded joint to fracture propagation increases (i.e., there is a reduction in $W_{3}$ and in the cleavage stress). Figure 6 shows the results for two bonded layers. Here, as the crack approaches the free surface, $k_{1}, k_{2}$ and $W_{2}^{\prime}$ become unbounded. For $\mu_{2}<\mu_{1}$, they all decrease as the crack approaches the interface, at $h_{3}=h_{2} k_{1}$ and $k_{2}$ are again discontinuous, and $w_{2}=2 w_{12} / c_{12}$ where $W_{12}$ may be evaluated from the solution of the interface crack problem (see Figure 9).

Figures 7-9 show some of the results for the interface crack problem. Here $k_{j}(\infty),(i=1,2)$ and $w_{23}(\infty)$ refer to the corresponding values for two bonded half planes with an interface crack $(i . e ., h=\infty)$. In the case of three materials (Figure 7) if $\mu_{3}<\mu_{2}, \mu_{3}<\mu_{1}$, for a given flaw size again the fracture resistance of the bond increases with decreasing layer thickness. As seen from Figures 8 and 9 , the opposite observation is valid if there is a free surface. Further results on the plane strain problem for a crack in layered materials may be found in $[3,4]$. The equivalent results for a penny-shaped crack are given in $[5,6]$. Similar results for an elastic fiber imbedded into an elastic matrix having perfect adhesion except for an axisymmetric interface crack are given in [8].

In the solutions given by the Figures $2-9$ the adhesive layer is treated as an elastic continuum, and it is assumed that the flaw is a crack. In some problems this may be a realistic model. In some other problems, however, these assumptions would not only complicate the analysis, they may not even be realistic. This 
would be the case, for example, in bonded materials in which the relative thickness of the adhesive is so small that the thickness variation of the stresses in the adhesive layer may be neglected. In such problems the adhesive may be approximated by a combination of shear and tension springs, which simplify the interface conditions considerably. Referring to the insert in Figure 10 , for two dimensional problems these conditions may be expressed as

$$
\begin{array}{ll}
\left(u_{2}-u_{1}\right) / h_{3}=\sigma_{x y} / \mu_{3}, & (y=0) \\
\left(v_{2}-v_{1}\right) / h_{3}=\sigma_{y y} / E_{3}, & (y=0)
\end{array}
$$

where $h_{3}, \mu_{3}, E_{3}$ are, respectively, the thickness, the shear modulus, and the Young's modulus of the adhesive, $u_{i}, v_{j},(i=$ $1,2)$ are the displacements and $\sigma_{x y}$, $\sigma_{y y}$ are the tractions on the surfaces of the two materials bonded through the adhesive.

For example, for the two half planes shown in Figure 10 , defining the adhesive stresses by

$$
\sigma_{y y}(x, 0)=p_{1}(x), \quad \sigma_{x y}(x, 0)=p_{2}(x)
$$

and using the solution for the elastic half plane [10], (22) may be expressed as

$$
\begin{aligned}
& \int_{-a}^{a} p_{2}(t) \log \left|\frac{t-x}{t}\right| d t+\gamma \int_{0}^{x} p_{1}(t) d t-b p_{2}(x)+e=0, \\
& \int_{-a}^{a} p_{1}(t) \log \left|\frac{t-x}{t}\right| d t-\gamma \int_{0}^{x} p_{2}(t) d t-c p_{1}(x)+d=0, \\
& \int_{-a}^{a} p_{1}(t) d t=P, \quad \int_{-a}^{a} p_{2}(t) d t=Q,
\end{aligned}
$$


where

$$
\begin{aligned}
& r=\gamma_{1} / \gamma_{2}, \quad b=h_{3} /\left(\mu_{3} \gamma_{2}\right), \quad c=h_{3} /\left(E_{3} \gamma_{2}\right), \\
& d=v_{2}(0) / \gamma_{2}, \quad e=u_{2}(0) / \gamma_{2}, \\
& \gamma_{1}=\frac{1}{4}\left(\frac{\kappa_{2}-1}{\mu_{2}}-\frac{\kappa_{1}-1}{\mu_{1}}\right), \quad \gamma_{2}=\frac{1}{4 \pi}\left(\frac{\kappa_{1}+1}{\mu_{1}}+\frac{\kappa_{2}+1}{\mu_{2}}\right),
\end{aligned}
$$

$p_{j}(x),(i=1,2)$ are the unknown functions and the unknown constants $u_{2}(0)$ and $v_{2}(0)$ are determined from the equilibrium conditions $(24 . c, d)$. The integral equations $(24 . a, b)$ have only logarithmic singularities; hence, the Fredholm theory is applicable and the functions $p_{i},(i=1,2)$ are bounded* .

Figure 10 shows the contact stresses for two elastic half planes bonded through an adhesive layer of width 2 a and thickness $h_{3}$. The figure also shows the results for the limiting case $h_{3}=0$ for which the closed form solution is given by [15]

$$
\begin{aligned}
& p_{1}(x)=\frac{p}{2 \pi} \frac{\alpha+1}{\alpha^{1 / 2}} \frac{1}{\left(a^{2}-x^{2}\right)^{1 / 2}} \cos \left[\omega \log \left(\frac{a-x}{a+x}\right)\right], \\
& p_{2}(x)=\frac{p}{2 \pi} \frac{\alpha+1}{\alpha^{1 / 2}} \frac{1}{\left(a^{2}-x^{2}\right)^{1 / 2}} \sin \left[\omega \log \left(\frac{a-x}{a+x}\right)\right], \quad(26 . a, b)
\end{aligned}
$$

where

$$
\alpha=\left(\kappa_{1} \mu_{2}+\mu_{1}\right) /\left(\kappa_{2} \mu_{1}+\mu_{2}\right), \quad \omega=\frac{1}{2 \pi} \log \alpha .
$$

It should be noted that, for the configuration given in

*From (24) it can be shown that at $x=\mp$ a even though $p_{i}(x)$, $(i=1,2)$ is bounded, the derivative $d p_{i} / d x$ has a logarithmic singularity, $i . e ., d p_{j} / d x \simeq K \log (a-x)$ for small values of $(a-x)$. 
Figure 10 if the relative thickness of the adhesive, $h_{3} / a$ is increased, the contact stresses become more "uniform" and their peak values decrease. Table 1 shows the values of the "stress concentration factors" for three different adhesive thicknesses. Further application of this concept to the stress analysis of the adhesive joints may be found in [17].

Table 1. Stress concentration factors

for two bonded half planes.

\begin{tabular}{cccc}
$h / a$ & 0.004 & 0.010 & 0.040 \\
\hline$\sigma_{y y}(a, 0) /(P / 2 a)$ & 4.240 & 2.900 & 1.672 \\
$\sigma_{x y}(a, 0) /(P / 2 a)$ & -0.7020 & -0.3322 & -0.1148
\end{tabular}

4. A CRACK TERMINATING AT AND GOING THROUGH AN INTERFACE

The solution of the problem of a crack propagating toward and terminating at a bimaterial interface was given in [7]. From the viewpoint of fracture propagation, in this problem the quantities of interest are the stress intensity factors, the power of the singularity, $\alpha$ (see equation (1)), and the angular distribution of the stresses around the crack tips. For the crack tip imbedded in the homogeneous medium $\alpha=1 / 2$ and the $\theta$-dependence of stresses are well-known (see, for example, [14]). In [7] a was obtained directly from the integral equations by using the function-theoretic approach. It may also be obtained by using the technique of the eigenfunction expansion [18].

The $\theta$-dependence of the stresses around the crack tip terminating at the interface is dependent on the elastic constants $\mu_{i}, \kappa_{i},(i=1,2)$ and $i s$ obtained from the solution of the 
problem [7]. Figures 11-13 show the results for two bonded semiinfinite planes where material 1 contains a semi-infinite crack perpendicular to and terminating at the interface. The plane is loaded by symmetric wedge forces $P$ applied to the crack surfaces at a distance $r_{0}$ from the crack tip.. In this problem for small values of $\left(r / r_{0}\right)$ the stresses may be expressed as

$$
\begin{aligned}
& \sigma_{i j}(r, \theta)=\frac{P}{r_{0}}\left(\frac{r_{0}}{r}\right) G_{i j}(\theta)+0\left[\left(r / r_{0}\right)^{\alpha}\right], \\
& \left(0<\alpha<1, \quad(i, j)=(r, \theta), 0 \leq \theta \leq \pi, \operatorname{Re}\left[\alpha_{2}\right]>0\right) .
\end{aligned}
$$

The functions $G_{i j}$ are shown in Figures $11-13$ where $m=\mu_{2} / \mu_{1}$ and the corresponding material combinations are given in Table 2 . The table also shows the stress intensity factor $k$ defined by

$$
k=\lim _{r \rightarrow 0} \sqrt{2} r^{\alpha} \sigma_{2 \theta \theta}(r, 0)
$$

which can be evaluated in closed form [7] and which, in the homogeneous medium, is given by

$$
k=\frac{P}{\pi}\left(\frac{2}{r_{0}}\right)^{1 / 2}
$$

Table 2. Stress intensity factors for semi-infinite crack.

\begin{tabular}{lcc} 
Material & $m=\mu_{2} / \mu_{1}$ & $\mathrm{k} /\left(\mathrm{P} / \sqrt{\mathrm{r}_{0}}\right)$ \\
\hline Boron-Epoxy & 0.0072 & 0.0511 \\
Aluminum-Epoxy & 0.0433 & 0.1197 \\
Aluminum-Aluminum & 1.00 & 0.4502 \\
Epoxy-Aluminum & 23.077 & 0.8403 \\
Epoxy-Boron & 138.462 & 0.8905 \\
Epoxy-Rigid & $\infty$ & 0.9015
\end{tabular}


In the general case (for symmetric loading) the asymptotic values of the stresses for small $r$ may be expressed as

$$
\begin{aligned}
\sigma_{i j}(r, \theta)=\frac{k}{\sqrt{2} r^{\alpha}} f_{i j}(\theta) & +0\left(r^{\alpha}\right), \\
\left(\operatorname{Re} \alpha_{2}\right. & >0, \quad i, j=r, \theta),
\end{aligned}
$$

where the functions $f_{i j}$ are related to $G_{i j}$ shown in Figures $11-13$ through

$$
f_{i j}(\theta)=G_{i j}(\theta) / G_{\theta \theta}(0), \quad(i, j=r, \theta) .
$$

In the case of a finite crack perpendicular to the interface, the stress intensity factors are shown in Figure 14. Here the external load is a uniform pressure, $p_{0}$ applied to the crack surface and the two materials considered are Aluminum $(\nu=0.3)$ and Epoxy $(\nu=0.35)$ (see Table 2 for modulus ratio). The power of the singularity, $\alpha$ for the crack tip terminating at the interface (i.e., $c=a_{0}$ ) is given in Table 3 . Note that, because of the variation in $k$, there is a slight difference between the values of a corresponding to plane strain and plane stress.

Table 3. Stress intensity factors and the power of singularity for a crack terminating at the interface.

$\mu_{2} / \mu_{1} \quad \begin{gathered}\text { Plane Strain } \\ \frac{k(a)}{p_{0} \sqrt{a_{0}}}\end{gathered} \frac{k(b)}{p_{0} \sqrt{a_{0}}} \quad \alpha \quad \frac{k(a)}{p_{0} \sqrt{a_{0}}} \cdot \frac{k(b)}{p_{0} \sqrt{a_{0}}}$

$\begin{array}{llllllll}23.077 & 0.3381 & 2.6237 & 0.8827 & 0.2890 & 4.1760 & 0.8789 \\ 0.0433 & 0.8258 & 0.0700 & 1.3552 & 0.8230 & 0.0744 & 1.3525\end{array}$


For this case Table 3 also shows the stress intensity factors at the crack tips $r=a=0$ and $r=b$ which are defined by

$$
\begin{aligned}
& k(a)=\lim _{r \rightarrow 0} \sqrt{2} r^{\alpha} \sigma_{2 \theta \theta}(r, 0), \\
& k(b)=\lim _{r \rightarrow b} \sqrt{2(r-b)} \sigma_{1 \theta \theta}(r, \pi) .
\end{aligned}
$$

Due to the deviation in a from $1 / 2$ as given in Table 3 , the limits of the curves $k(a)$ shown in Figure 14 as $c \rightarrow a_{0}$ are 0 and $\infty$ for $\mu_{1}<\mu_{2}$ and $\mu_{1}>\mu_{2}$, respectively.

To solve the problem of a crack going through the interface, first the problem of two cracks (one in each material) perpendicular to the interface is considered. The problem is again formulated by using the Mellin transform. In the resulting system of singular integral equations the input functions are the crack surface tractions. For the case in which the two cracks are imbedded into the adjacent homogeneous media, the kernels of the integral equations have only Cauchy type singularities. Hence the solution of the problem is rather straightforward. When the inner crack tips go to the interface, the cracks join and become a through crack. In this limiting case, even though the integral equations remain unchanged, the singularities of the kernels become of the generalized Cauchy type. This means that $r=0$ (as well as $r=b_{1}$ and $r=b_{2}$ ) is an irregular point around which the solution will have a singularity of the form $r^{-\alpha},(0<\alpha<1 / 2)^{*}$.

*This is the case for most of the practical material combinations. However, for some unusual combinations the stresses at $r=0$ may be bounded or may have only a logarithmic singularity (see [19] for a thorough discussion). 
The system of characteristic equations giving $\alpha$ and the powers of the singularity at the two (outside) ends of the crack is obtained by applying the function-theoretic method directly to the system of singular integral equations.

In the examples considered in this paper, it is assumed that the material is subjected to tensile loads parallel to the interface and sufficiently away from the location of the crack. For the perturbation problem it may then be assumed that the crack surface tractions are uniform pressures related by

$$
p_{1} / p_{2}=E_{1} / E_{2}
$$

where the subscripts 1 and 2 refer to materials $1\left(\frac{\pi}{2}<\theta<\frac{3 \pi}{2}\right)$ and $2\left(-\frac{\pi}{2}<\theta<\frac{\pi}{2}\right)$, respectively.

The stress intensity factors for two cracks imbedded into the adjacent bonded $p l a n e s$ are shown in Figure 15 . In this example Material 1 is aluminum, Material 2 is Epoxy, the crack lengths $2 l_{1}=b_{1}-a_{1}, 2 l_{2}=b_{2}-a_{2}$ are equal and fixed, $a_{2}$ is constant, $a_{2}=2 l_{2}$ is the length unit, and the variable is the distance of the first crack to the interface, or $\left(b_{1}+a_{1}\right) / 2 a_{2}$. In the special case of the homogeneous medium containing two collinear cracks of equal lengths and subjected to a uniform pressure $p_{0}$ on the crack surfaces, the stress intensity factors are given by [20]

$$
\frac{k(a)}{p_{0} \sqrt{l}}=\frac{\left(b^{2} \frac{E(m)}{k(m)}-a^{2}\right) \sqrt{2}}{(b-a)[a(b+a)]^{1 / 2}},
$$




$$
\begin{aligned}
& \frac{k(b)}{p_{0} \sqrt{l}}=\frac{\sqrt{2} b^{2}[1-E(m) / K(m)]}{(b-a)[b(b+a)]^{7 / 2}}, \\
& \quad\left(2 l=b-a, \quad m=1-a^{2} / b^{2}\right),
\end{aligned}
$$

where $2 \mathrm{a}$ and $2 \mathrm{~b}$ are the distances between inner and outer tips of the cracks, respectively. For example, for $b=2 a$ we obtain

$$
k(a)=1.01762 p_{0} e^{1 / 2}, \quad k(b)=1.01249 \mathrm{p}_{0} e^{1 / 2} .
$$

Figures 16-22 show some of the results obtained for the material combination Aluminum-Epoxy. The stress intensity factors shown in these figures are defined as follows:

$$
\begin{aligned}
& k_{1}=\lim _{r \rightarrow b_{1}} \sqrt{2\left(r-b_{1}\right)} \sigma_{1 \theta \theta}(r, \pi), \\
& k_{2}=\lim _{r \rightarrow b_{2}} \sqrt{2\left(r-b_{2}\right)} \sigma_{2 \theta \theta}(r, 0), \\
& k_{\theta}=\lim _{r \rightarrow 0} r^{\alpha} \sigma_{1 \theta \theta}\left(r, \frac{\pi}{2}\right), \\
& k_{r}=\lim _{r \rightarrow 0} r^{\alpha} \sigma_{1 r \theta}\left(r, \frac{\pi}{2}\right) .
\end{aligned}
$$

The factors $k_{\theta}$ and $k_{r}$ are a measure of the contact stresses around $r=0$ and may be used in studying the question of debonding crack initiation. The stress intensity factors shown in the figures are normalized with respect to $p_{1} \ell^{1 / 2}, p_{2} \ell^{1 / 2}$, and $p_{1} e^{\alpha}$, where $2 l$ is the total crack length.

In Figures 16-18 material 1 is aluminum, material 2 is epoxy, the penetration of the crack into 1 is fixed and is taken as the length unit, i.e., $b_{1}=1$, and the crack length $2 l$ (or the crack 
penetration into the second medium) is the variable. For plane strain case and for the material pair under consideration the power of singularity at $r=0$ (i.e., for two bonded quarter planes) is found to be $\alpha=0.273692$. In the limiting case of $\ell=0.5, b_{2}=0$ and the problem reduces to that of a crack terminating at the interface for which the results are given in Table 3. Since at $r=0$ the power of singularity for this limiting case (i.e., $\alpha=0.8258$ ) is greater than $1 / 2$, as seen from Figures 16 and 17 , the stress intensity factors $k_{2}, k_{\theta}$, and $k_{r}$ become unbounded as $\ell \rightarrow 0.5$. For the same material combination the crack surface displacement is shown in Figure 18 . Here the functions $u_{1 \theta}(r, \pi),\left(0<r<b_{1}\right)$ and $u_{2 \theta}(r, 0),\left(0<r<b_{2}\right)$ are plotted against the dimensionless variables $\xi_{1}$ and $\xi_{2}$ defined by

$$
\xi_{1}=2 \frac{r}{b_{1}}-1, \quad \xi_{2}=2 \frac{r}{b_{2}}-1 \text {. }
$$

As seen from the figure, because of the singularity at $r=0$, it turns out that the displacement has an unbounded derivative at $r=0$ as we 11 as at $r=b_{i},(i=1,2)$.

Figures 19 and 20 show the results analogous to that given by Figures 16 and 17 , the difference being that here material 1 is epoxy and material 2 is aluminum. In the limiting case of $\ell=1 / 2$ the power of the singularity is $\alpha=0.3381$ (see Table 3 ), which is less than $1 / 2$ and is greater than the power of the singularity $\alpha=0.273692$ at $r=0$ for $\ell .>1 / 2$. Thus, as seen from Figures 19 and 20 , as $\ell$ goes to $1 / 2, k_{2}$ goes to zero and $k_{r}$ and $k_{\theta}$ become unbounded. 
Further results for the material Combination Aluminum (1) Epoxy (2) are shown in Figures 21 and 22. In this case the total crack length $2 l$ is constant and the crack eccentricity $c$ is selected as the variable.

\section{PENNY-SHAPED CRACK IN A FILAMENT-REINFORCED ELASTIC MATRIX}

In filament-reinforced composites the spacing and the orientation of the filaments are generally random. However, in some cases it is desirable and, to a certain extent, possible to control the orientation of the filaments in the matrix. In this Section we will describe some of the results of a study on the stress distribution in a filament-reinforced elastic matrix containing a penny-shaped crack [21]. It will be assumed that (a) the matrix is "sparsely" reinforced by a finite number of filaments which are symmetrically distributed around the crack and are oriented perpendicular to the plane of the crack, (b) $z=0$ is a plane of symmetry for the external loads and for the geometry, and $(c)$ the filament radius $r_{0}$ is small compared to its length 2c. Under these assumptions the problem can be formulated in terms of an integral equation with essentially the interface shear stress between the filament and the matrix as the unknown function. The main interest in this problem is in the evaluation of (a) the stress intensity factor at the crack periphery as a function of $\theta,(0<\theta<2 \pi)$, (b) the contact stress between the filament and the matrix as a function of $z$, $(-c<z<c)$, and $(c)$ the stress concentration factor in the filament. 
Figures 23-25 show the $\theta$-dependence of the stress intensity factor, $K_{1}$. In the examples given in this section it is assumed that the medium is subjected to uniaxial tension in z-direction, $\sigma_{z z}=\sigma_{0}$, away from the location of the crack and the filaments. Figure 23 shows the variation of the stress intensity factor in the case of a single filament for various values of filament-tomatrix modulus ratio, $E_{f} / E$ and for filament-to-crack center distance, $b$. It is seen that the effect of the filament on the stress intensity factor becomes more significant as the distance $b$ decreases and as the moduius ratio $E_{f} / E$ increases.

The effect of the number $L$ of symmetrically located (identical) filaments on the stress intensity factor for a given modulus ratio, $\left(E_{f} / E\right)=100$ is shown in Figure 24 . The figure indicates that for $b \geq 1.5$ and $L \geq 6$ the $\theta$-dependence of the stress intensity factor may be neglected. Figure 25 shows the effect of the modulus ratio, $E_{f} / E$ on $K_{j}$ for $L=2$ and $L=4$.

Finally, the effect of the modulus ratio on the maximum tensile stress in the filament is shown in Figure 26. Here $\bar{\sigma}_{c} f(0)$ is the stress in the filament at $z=0$ and $\sigma_{0}$ is the stress in the matrix away from the crack-filament region. For large values of the filament length $2 c$ and the distance $b$ we have $\left(\bar{\sigma}_{c}^{f}(0) / \sigma_{0}\right) \simeq\left(E_{f} / E\right)$. For comparison the figure also shows the stress at the same location in the absence of any filaments (i.e., for $E_{f}=E$ ). obtained from the axisymmetric penny-shaped crack solution [22]. Further results on this problem may be found in [21]. An approximate solution of a special case of the 
problem is given in [23] where the $\theta$-dependence of the.stress state in the composite medium is neglected.

\section{INCLUSION PROBLEMS IN BONDED DISSIMILAR MATERIALS}

In some composites material imperfection may be in the form of an elastic inclusion rather than a crack. In this case it may be expected that due to the high stress concentration, the neighborhood of inclusion borders would be the likely location at which the fracture nucleation would occur. To give some idea about the nature of the problem, in this section we will discuss the solution of an idealized plane inclusion problem. It will be assumed that the inclusion is oriented parallel to the interface of two bonded elastic half planes and the thickness of the inclusion is very small compared to its lateral dimensions.

Thus, analytically, it can be approximated by a singular surface across which the displacement vector is continuous and the stress.. vector suffers a discontinuity. The formulation of the problem then leads to a system of singular integral equations.

In the case of a perfectly rigid inclusion, the solution of the plane problem gives the stress state in the close neighborhood of an end point as follows (see [9]):

$$
\begin{aligned}
& \sigma_{y y}(r, \theta)=\frac{1}{(2 r)^{1 / 2}}\left(k_{1} \cos \frac{\theta}{2}+\frac{k+1}{k-1} k_{2} \sin \frac{\theta}{2}\right)+0\left(r^{1 / 2}\right), \\
& \sigma_{x y}(r, \theta)=\frac{1}{(2 r)^{1 / 2}}\left(-\frac{\kappa+1}{k-1} k_{1} \sin \frac{\theta}{2}+k_{2} \cos \frac{\theta}{2}\right)+0\left(r^{1 / 2}\right), \\
& \sigma_{x x}(r, \theta)=\frac{1}{(2 r)^{1 / 2}}\left(-\frac{3+k}{k-1} k_{1} \cos \frac{\theta}{2}+\frac{3-k}{k-1} k_{2} \sin \frac{\theta}{2}\right)+0\left(r^{1 / 2}\right),
\end{aligned}
$$




$$
r e^{i \theta}=x-a+i y, \quad(-\pi<\theta<\pi),
$$

where $a$ is the half-length of the inclusion. If the distance, $h$ from the inclusion to the interface becomes zero (i.e., if the inclusion is located on the interface), the problem can be solved in closed form [9]. In this case, similar to the interface crack problems, the stresses at the end point have the well-known oscillating singularity; hence, at $h=0$, the stress intensity factors $k_{1}$ and $k_{2}$ (considered as functions of $h$ ) are discontinuous.

Perhaps a more realistic model for the inclusion would be an elastic layer with known extensional stiffness. Since the relative thickness of the inclusion is assumed to be very small, its bending stiffness will also be very small and may be neglected. For this "elastic membrane" type of inclusion the stress state around the end point may be expressed as [9]

$$
\begin{aligned}
& \sigma_{y y}(r, \theta)=\frac{k}{(2 r)^{1 / 2}} \cos \frac{\theta}{2}+0\left(r^{1 / 2}\right), \\
& \sigma_{x y}(r, \theta)=\frac{k}{(2 r)^{1 / 2}} \sin \frac{\theta}{2}+0\left(r^{1 / 2}\right), \\
& \sigma_{x x}(r, \theta)=-\frac{3 k}{(2 r)^{1 / 2}} \cos \frac{\theta}{2}+0\left(r^{1 / 2}\right) .
\end{aligned}
$$

Here as $h \rightarrow 0$, unlike the problem of rigid inclusion, the integral equation does not change its character and remains as a singular integral equation of the first kind. Hence the singular behavior of the stress state maintains its simple $r^{-1 / 2}$ character (see Figure 28). 
Figures 27-29 show some of the results obtained for rigid and elastic inclusion. In these examples it is assumed that the medium is subjected to symmetric loading away from the location of the inclusion. Thus the displacements in the medium without the inclusion and at the same location as the inclusion may be expressed as

$$
u_{0}=-\varepsilon_{0} x, \quad v_{0}=0 .
$$

The constant $k_{0}$ shown in the figures which is used to normalize the stress intensity factors is defined by

$$
k_{0}=\mu_{2} \varepsilon_{0} a^{1 / 2}\left(\kappa_{2}-1\right) / \kappa_{2},
$$

where $\mu_{2}$ and $\kappa_{2}$ are the elastic constants of the medium surrounding the inclusion.

Figure 27 shows the results for a rigid inclusion in two bonded elastic half planes and that in a half plane. Similar results for an elastic inclusion are shown in Figure 28 . Figure 28 also shows the stress intensity factor for an inextensible inclusion with zero bending stiffness. The values of $k$ for $h \rightarrow 0$ are obtained from the interface inclusion problem or from the problem of a stiffener bonded to an elastic half plane [24]. The figure indicates that the stress intensity factor is highly dependent on the stiffness of the inclusion.

In this problem too one could determine the orientation of the probable fracture nucleation by considering the cleavage stress $\sigma_{\theta \theta}$ as a function of $\theta$. For example, for the elastic 
inclusion problem from (38) we obtain

$$
\begin{aligned}
\sigma_{\theta \theta}(r, \theta)=\frac{k}{(2 r)^{1 / 2}} & \left(2 \cos \frac{\theta}{2} \cos 2 \theta-\cos \frac{\theta}{2}-\sin \frac{\theta}{2} \sin 2 \theta\right) \\
& +0\left(r^{1 / 2}\right), \quad(-\pi<\theta<\pi) .
\end{aligned}
$$

From $\left(\partial \sigma_{\theta \theta} / \partial \theta\right)=0$ we find $\theta=0^{\circ}, 74.3^{\circ}, 151^{\circ}$. The angular variation of $\sigma_{\theta \theta}$ is shown in Figure 29 . It is seen that if the medium is loaded perpendicular to the inclusion the fracture initiation will most likely be collinear with the inclusion with possible secondary radial cracks along $\theta= \pm 151^{\circ}$. On the other hand if the medium is loaded parallel to the inclusion the fracture would most likely nucleate along (roughly) $\theta= \pm 74.3^{\circ}$ radial lines.

\section{REFERENCES}

1. B. M. Malyshev and R. L. Salganik, "The strength of adhesive joints using the theory of fracture", Int. J. Fracture Mechanics, vo1. 1, p. 114 (1965).

2. F. Erdogan, "Bonded dissimilar materials containing cracks parallel to the interface", Engineering Fracture Mechanics, vol. 3, p. 231 (1971).

3. F. Erdogan and G. D. Gupta, "Stress analysis of multilayered composites with a flaw", Int. J. Solids, Structures, vol. 7, p. 39 (1971).

4. F. Erdogan and G. D. Gupta, "Layered composites with an interface flaw", Int. J. Solids, Structures, vol. 7, p. 1089 (1971).

5. K. Arin and F. Erdogan, "Penny-shaped crack in an elastic layer bonded to dissimilar half spaces", Int. J. Engng. sci., vol. 9, p. 213 (1971).

6. F. Erdogan and K. Arin, "Penny-shaped interface crack between an elastic layer and a half space"., Int. J. Engng. Sci., vol. 10, p. $115(1972)$. 
7. T. S. Cook and F. Erdogan, "Stresses in bonded materials with a crack perpendicular to the interface", Int. J.

Engng. Sci. (to appear, 1972).

8. F. Erdogan and T. Özbek, "Stresses in fiber-reinforced composites with imperfect bonding", J.Appl. Mech., vol. 36, p. 865, Trans. ASME, Series E (1969).

9. F. Erdogan and G. D. Gupta, "Stresses near a flat inclusion in bonded dissimilar materials", Int. J. Solids, Structures (to appear, 1972).

10. N. I. Muskhelishvili, Singular Integral Equations, P. Noordhoff, Groningen, The Netherlands (1953).

11. F. Erdogan, "Approximate solution of systems of singular integral equations", SIAM J. Appl. Math., vol. 17, p. 1041 (1969).

12. F. Erdogan and G. D. Gupta, "On the numerical solution of singular integrai equations", Quarterly of Applied Mathematics, p. 525 (1972).

13. F. Erdogan, G. D. Gupta, and T. S. Cook, "Numerical solution of singular integral equations" in Methods of Analysis of Crack Problems, G. C. Sih, editor ( to appear, 1973).

14. M. L. Williams, "On the stress distribution at the base of a stationary crack", J. Appl. Mech., vol. 26, p. 109, Trans. ASME (1957).

15. F. Erdogan, "Stress distribution in a nonhomogeneous, elastic piane with cracks", J. Appl. Mech., vol. 30, p. 232, Trans. ASME (1963).

16. F. Erdogan and G. C. Sih, "On the crack extension in plates under plane loading and transverse shear", J. Basic Engng., Trans. ASME, vol. 85, Series D, p. 519 (1963).

17. F. Erdogan and M. Ratwani, "Stress distribution in bonded joints", J. Composite Materials, vol. 5, p. 378 (1971).

18. A. R. Zak and M. L. Williams, "Crack point stress singularities at a bi-material interface", J. Appl. Mech., vol. 30, p. 142, Trans. ASME (1963).

19. D. B. Bogy, "Two edge-bonded elastic wedges of different materials and wedge angles under surface tractions", $J$. Appl. Mech., vol. 38, p. 377, Trans. ASME (1971).

20. F. Erdogan, "On the stress distribution in plates with col1 inear cuts under arbitrary loads", Proc. of the 4 th U.S. Nat. Congress of Applied Mechanics, vol. 1, p. 547, ASME, New York (1962). 
21. A. H. Pacella, "A penny-shaped crack in a filamentreinforced matrix", Ph.D. Dissertation, Lehigh University (1970).

22. I. N. Sneddon, Fourier Transforms, McGraw-Hi11, New York (1951).

23. J.P. Romualdi and G. B. Batson, "Mechanics of crack arrest in concrete", J. Engng. Mech., Proc. ASCE, vol. 89, p. 147 (1963).

24. F. Erdogan and G. D. Gupta, "The problem of an elastic stiffener bonded to a half plane", J. Appl. Mech., vol. 38, p. 937, Trans. ASME (1971). 


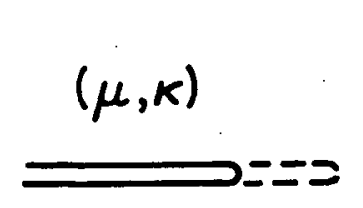

(a)

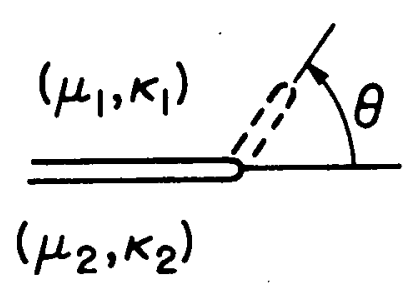

(d)

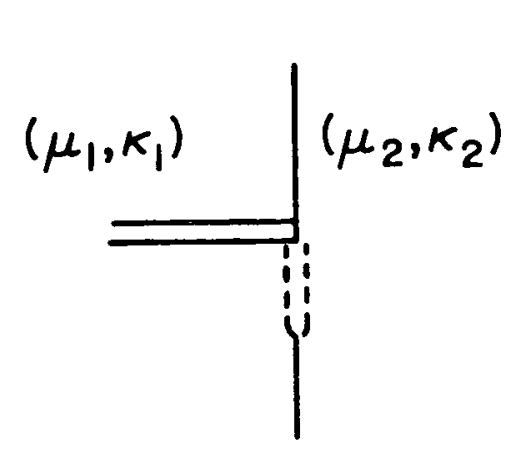

(g)

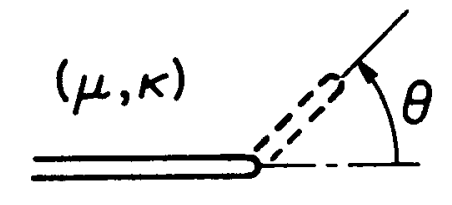

(b)

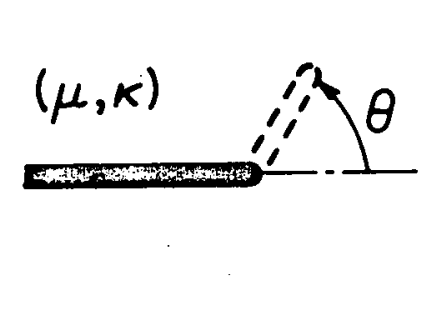

(e)

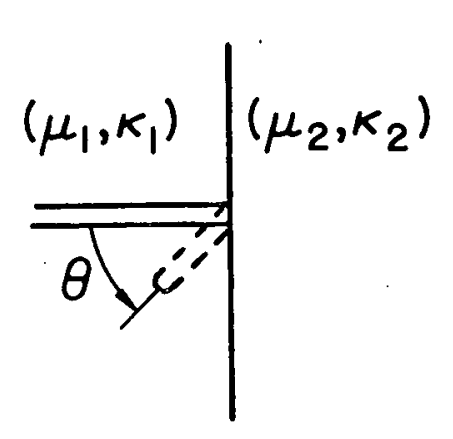

(h)

$$
\frac{\left(\mu_{1}, k_{1}\right)}{\left(\mu_{2}, \kappa_{2}\right)}==2
$$

(c)

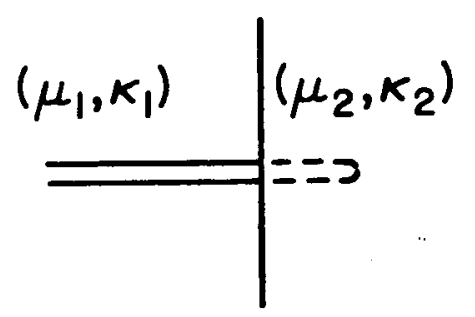

(f)

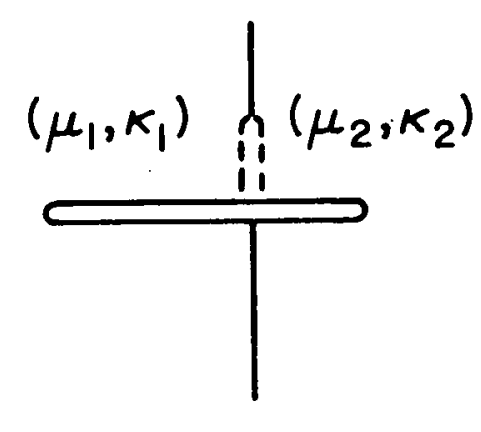

(i)

Fig. 1. Various possible modes of crack propagation in composite materials.

32 


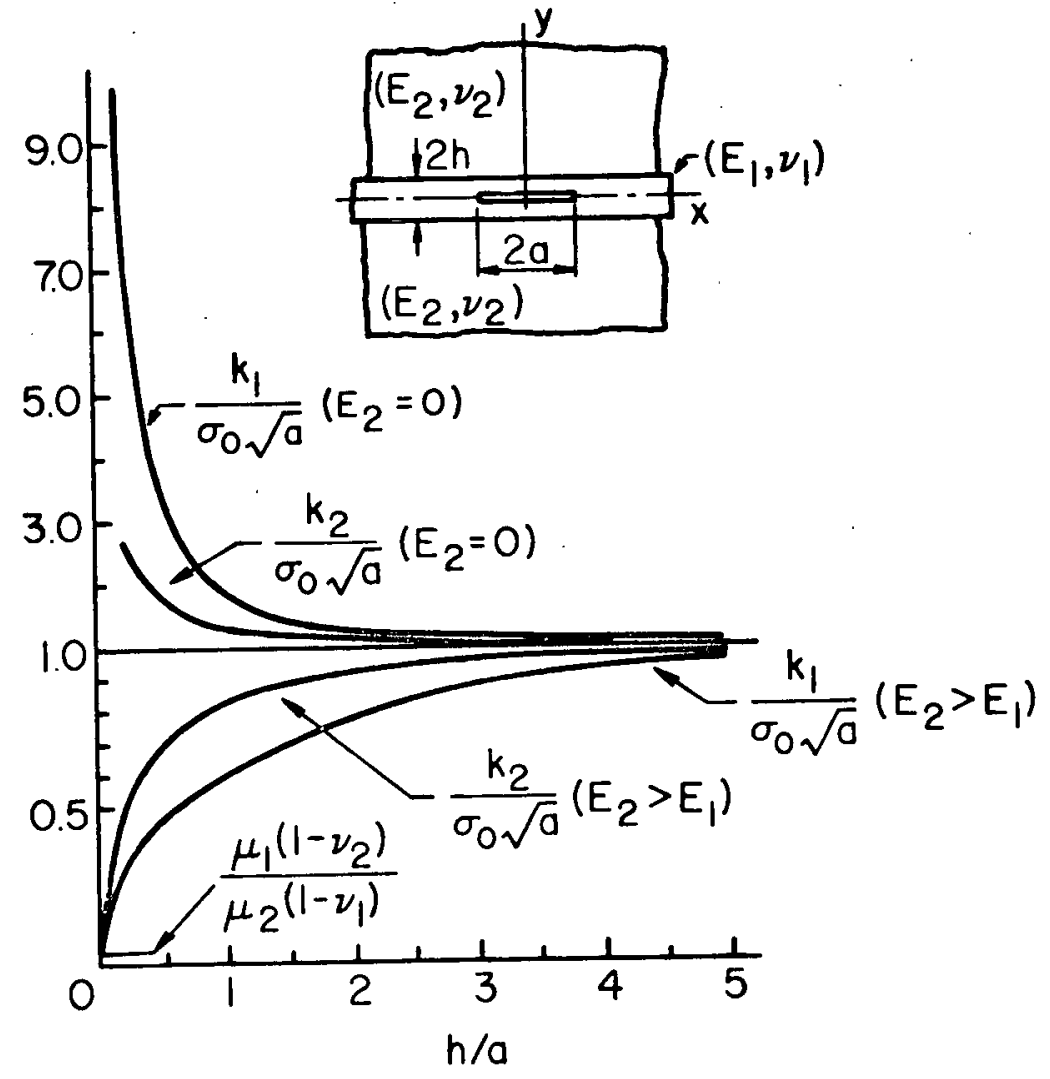

Fig. 2. Stress intensity factors in two similar half planes bonded through an elastic layer. $\quad\left(E_{1}=4.5 \times 10^{5}\right.$ psi, $v_{1}=0.35, E_{2}=10^{7} \mathrm{psi}, v_{2}=0.3$; external loads: $\sigma_{y y}(x, 0)=-\sigma_{0}$ or $\left.\sigma_{x y}(x, 0)=-\tau_{0},-a<x<a\right)$. 


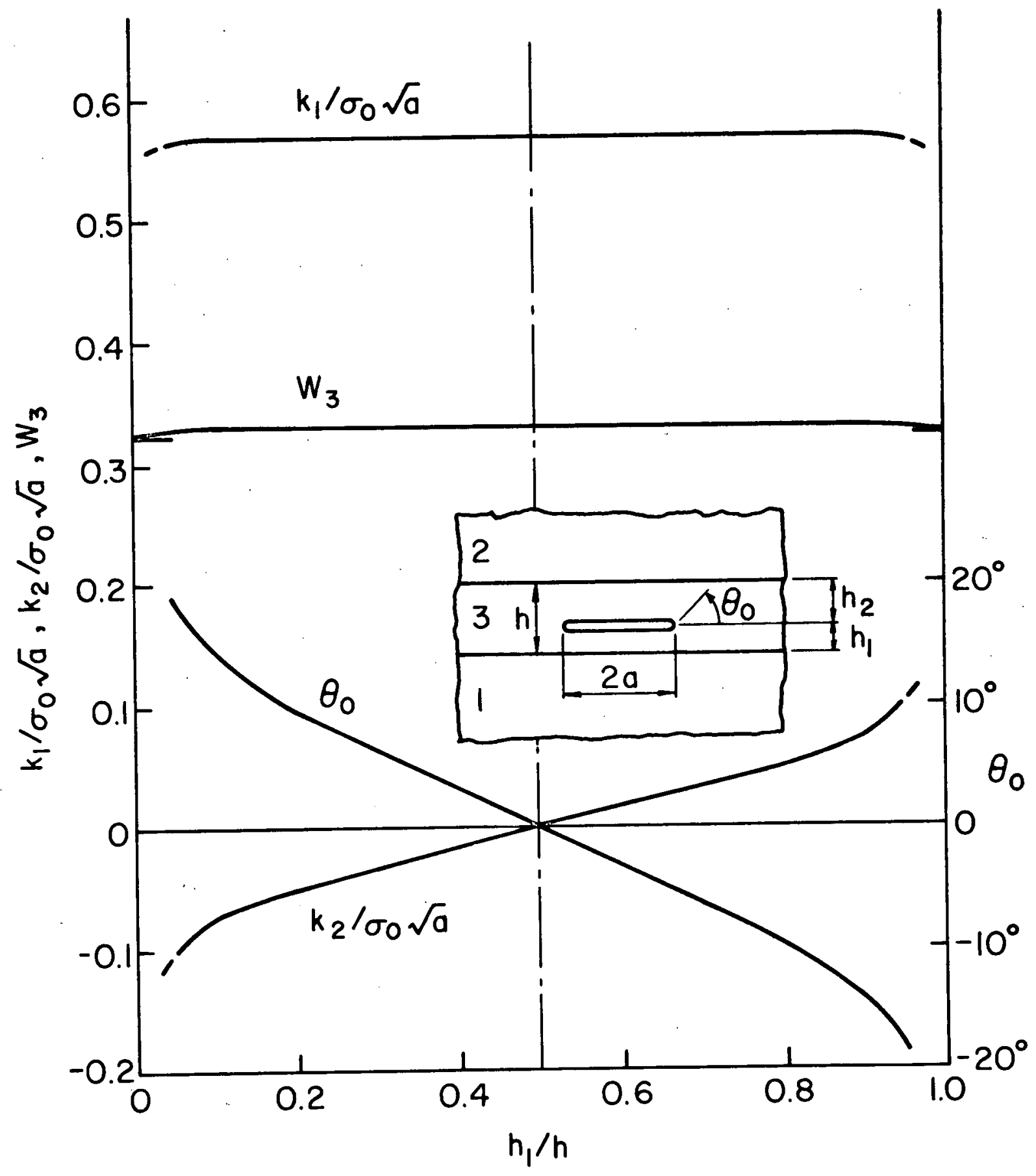

Fig. 3. Two similar materials bonded through a cracked layer. $\left(E_{1}=E_{2}=10^{7}\right.$ psi, $v_{1}=v_{2}=0.3, E_{3}=4.5 \times 10^{5}$ psi, $v_{3}=0.35 ; h=2 a$; external load: $\sigma_{y y}(x, 0)=-\sigma_{0}$, $-a<x<a$ ). 


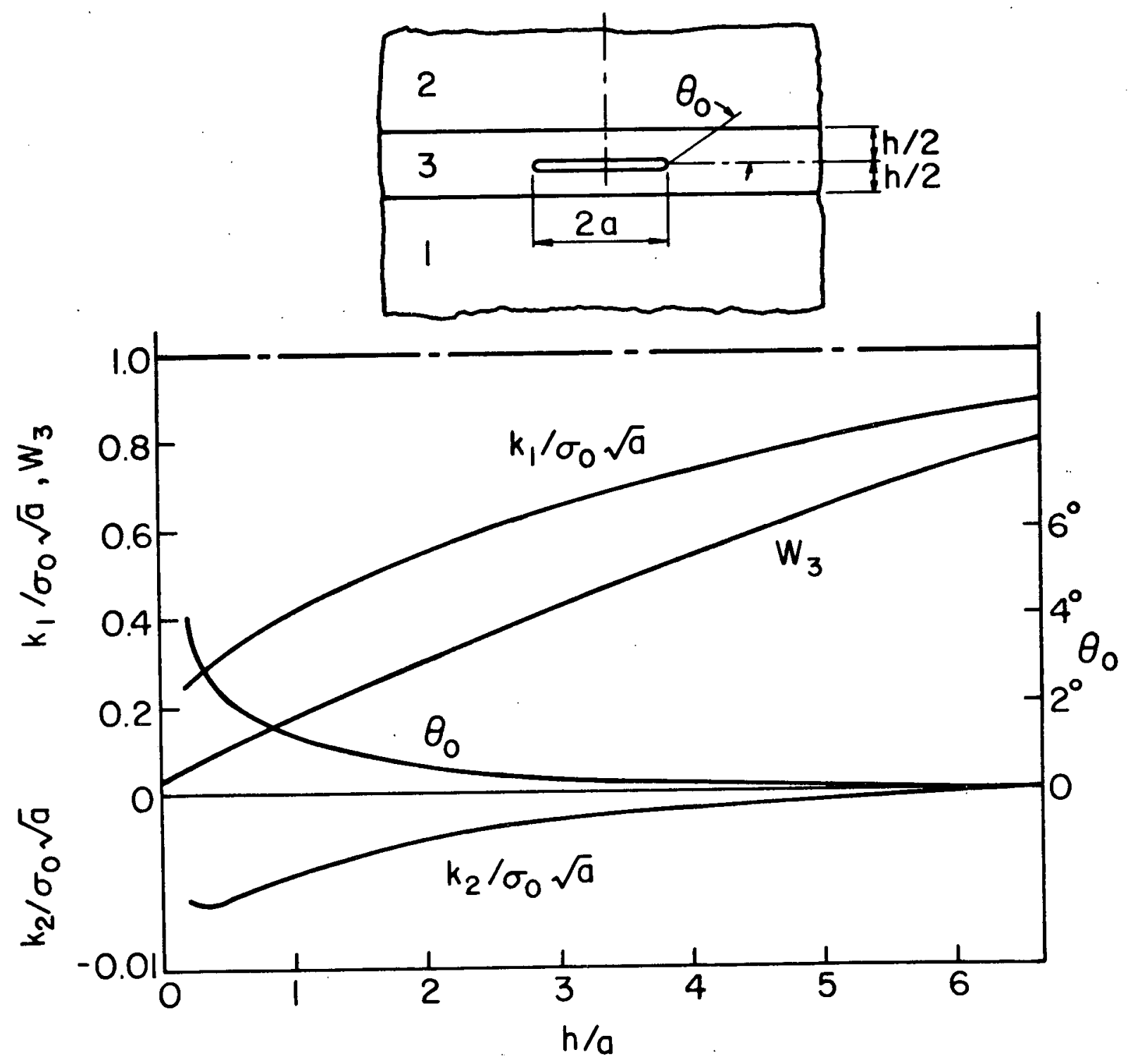

Fig. 4. Crack in the layer bonding two different half planes. (Materials: 1: Stee1, 2: Aluminum, 3: Epoxy.) 


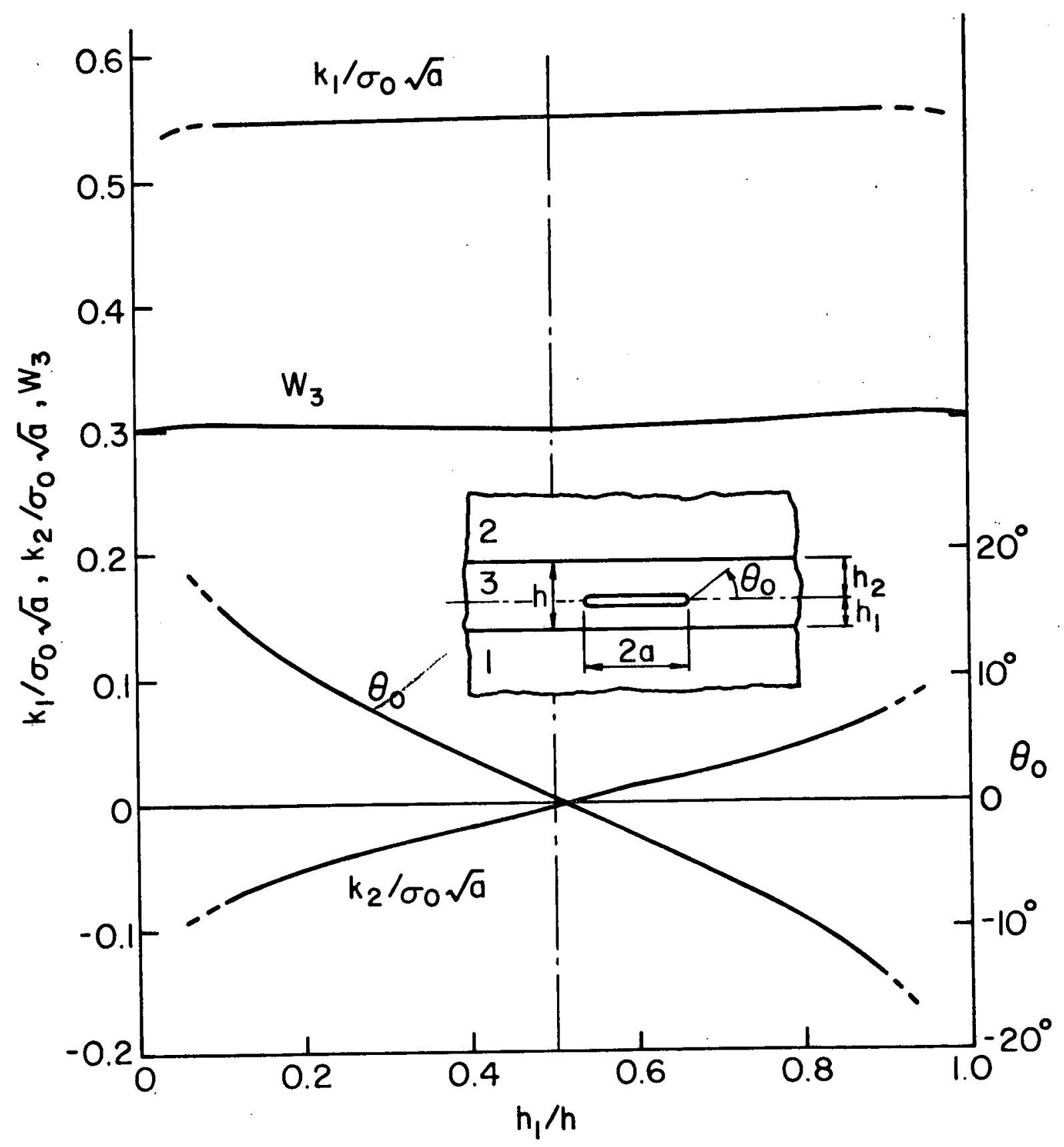

Fig. 5. Crack in the layer bonding two different half planes. $(h=2 a$, Materials: same as in.Fig. 4.) 


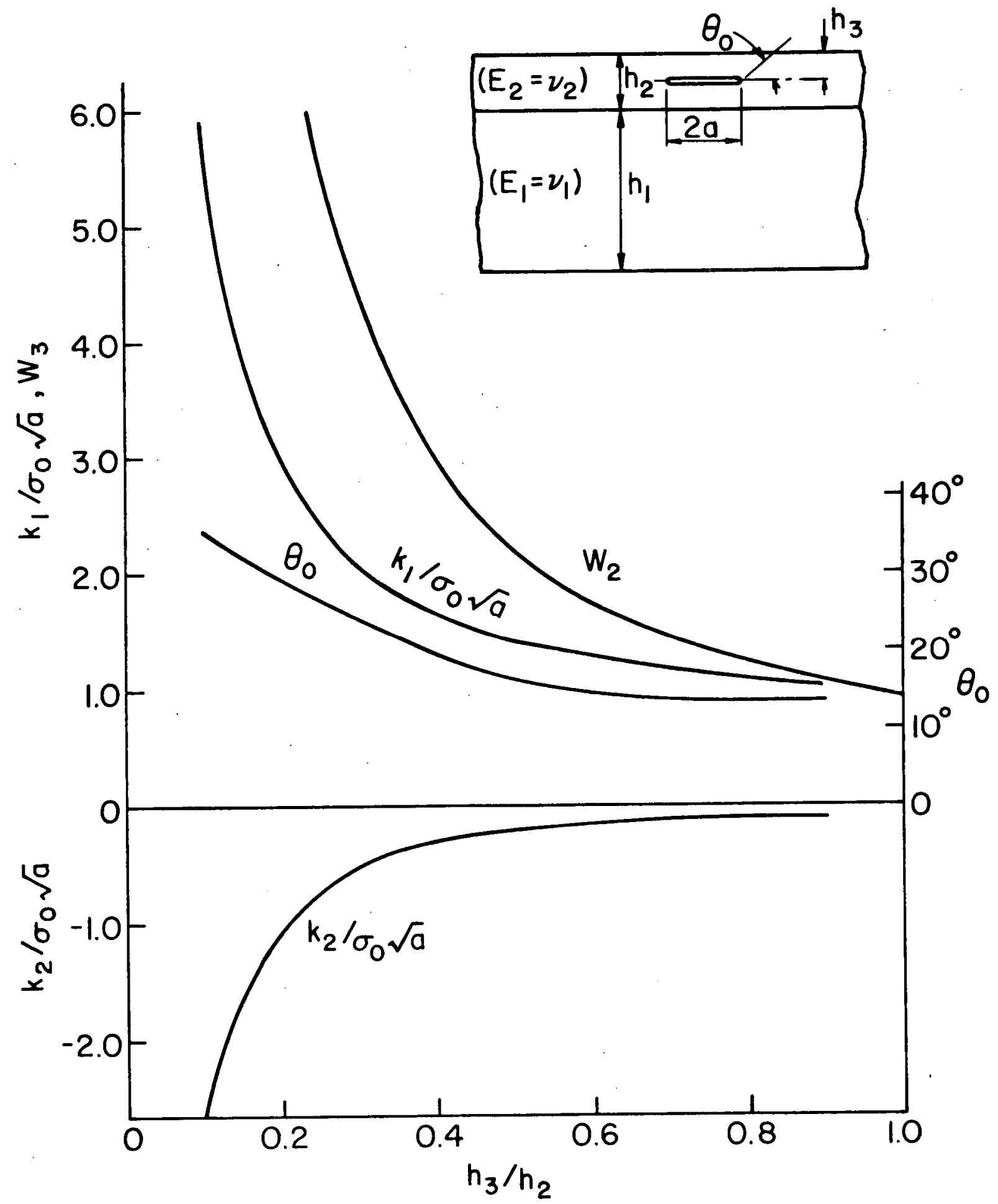

Fig. 6. Two bonded elastic layers containing a crack. (Materials: 1: Steel, 2: Aluminum; $h_{2}=2 a, h_{1}=3 h_{2}$; external load: crack surface pressure $\sigma_{n} \cdot$ ) 


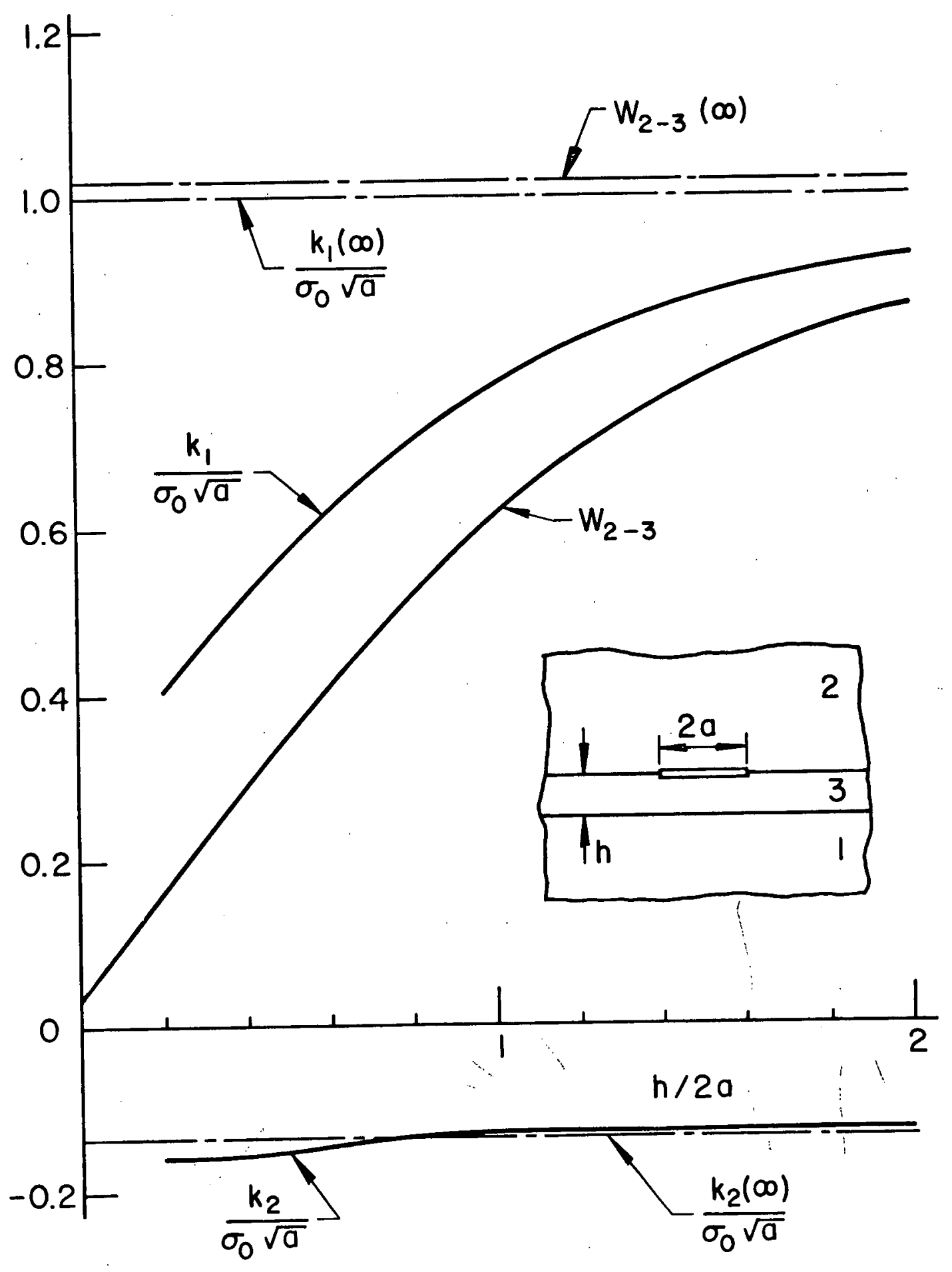

Fig. 7. Interface crack in two half planes bonded through an elastic layer. $k_{1}, k_{2}$ : stress intensity factors, $W_{23}$ strain energy release rate. (Materials: 1: Steel, 2: Aluminum, 3: Epoxy; load: crack surface pressure.) 


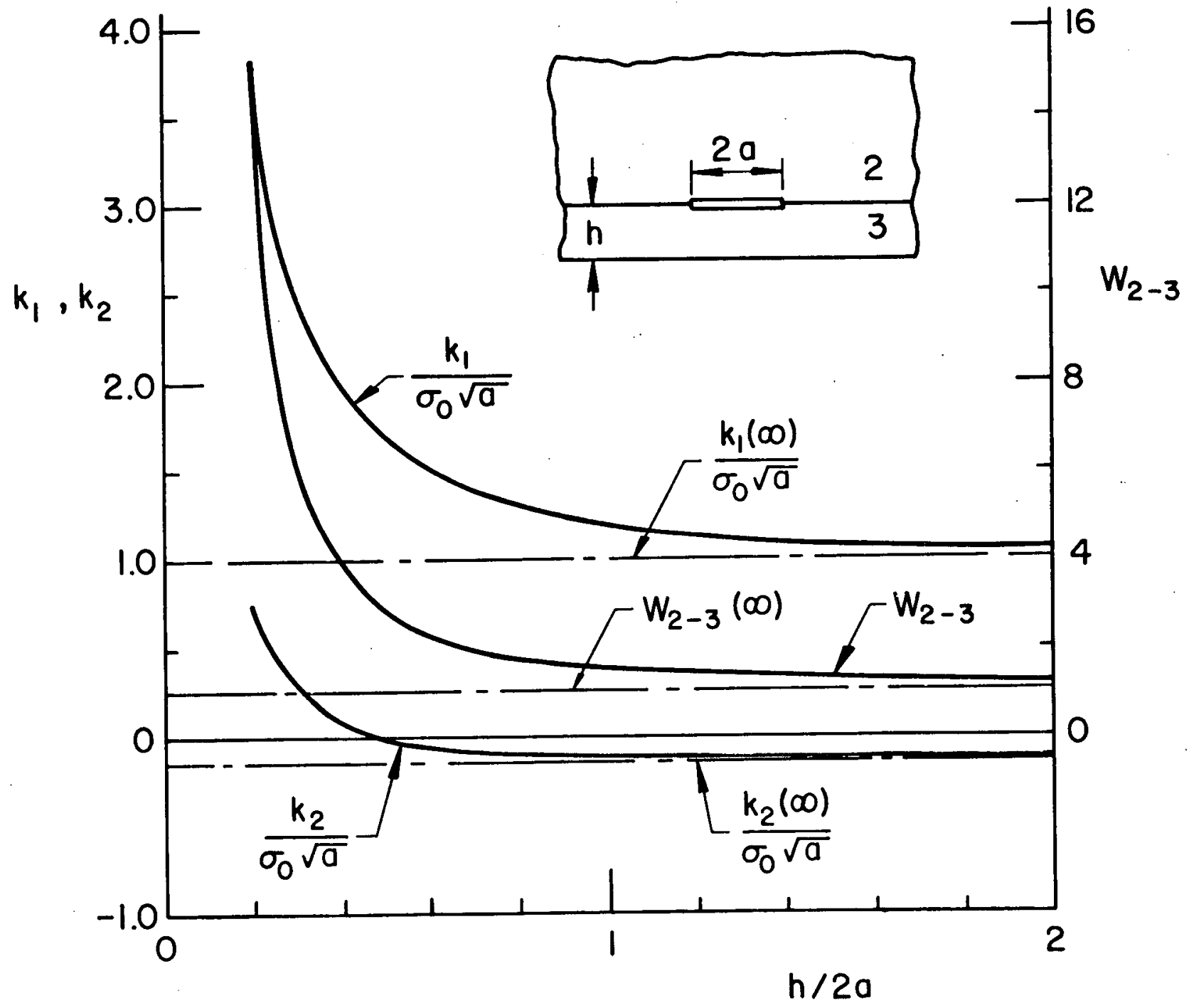

Fig. 8. Interface crack between a layer and a half plane. (Materials: 2: Aluminum, 3: Epoxy.) 


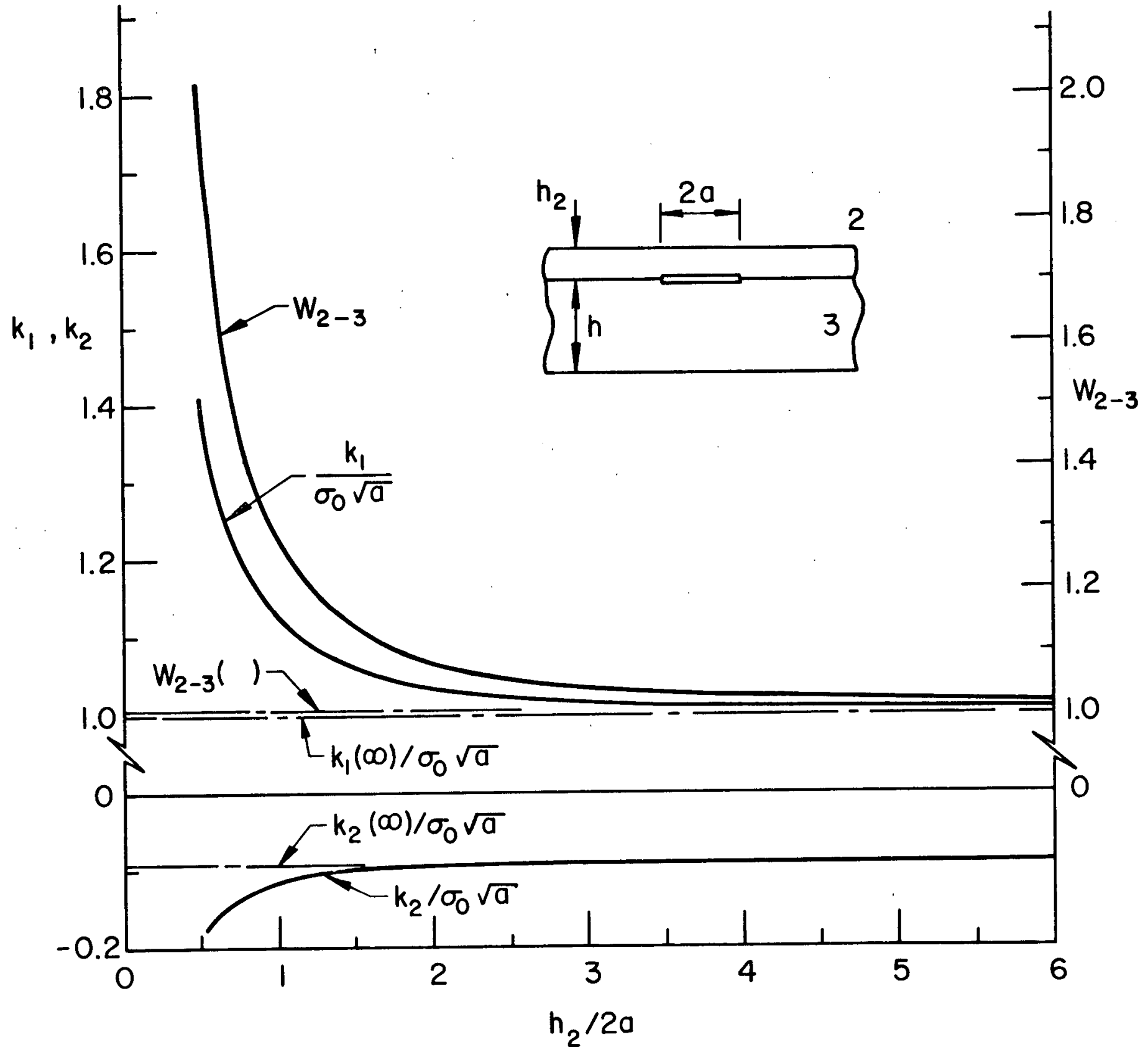

Fig. 9. Interface crack between two bonded elastic layers. ( $h=3 h_{2}$; Materials: 2: Steel, 3: Aluminum; load: crack surface pressure.) 


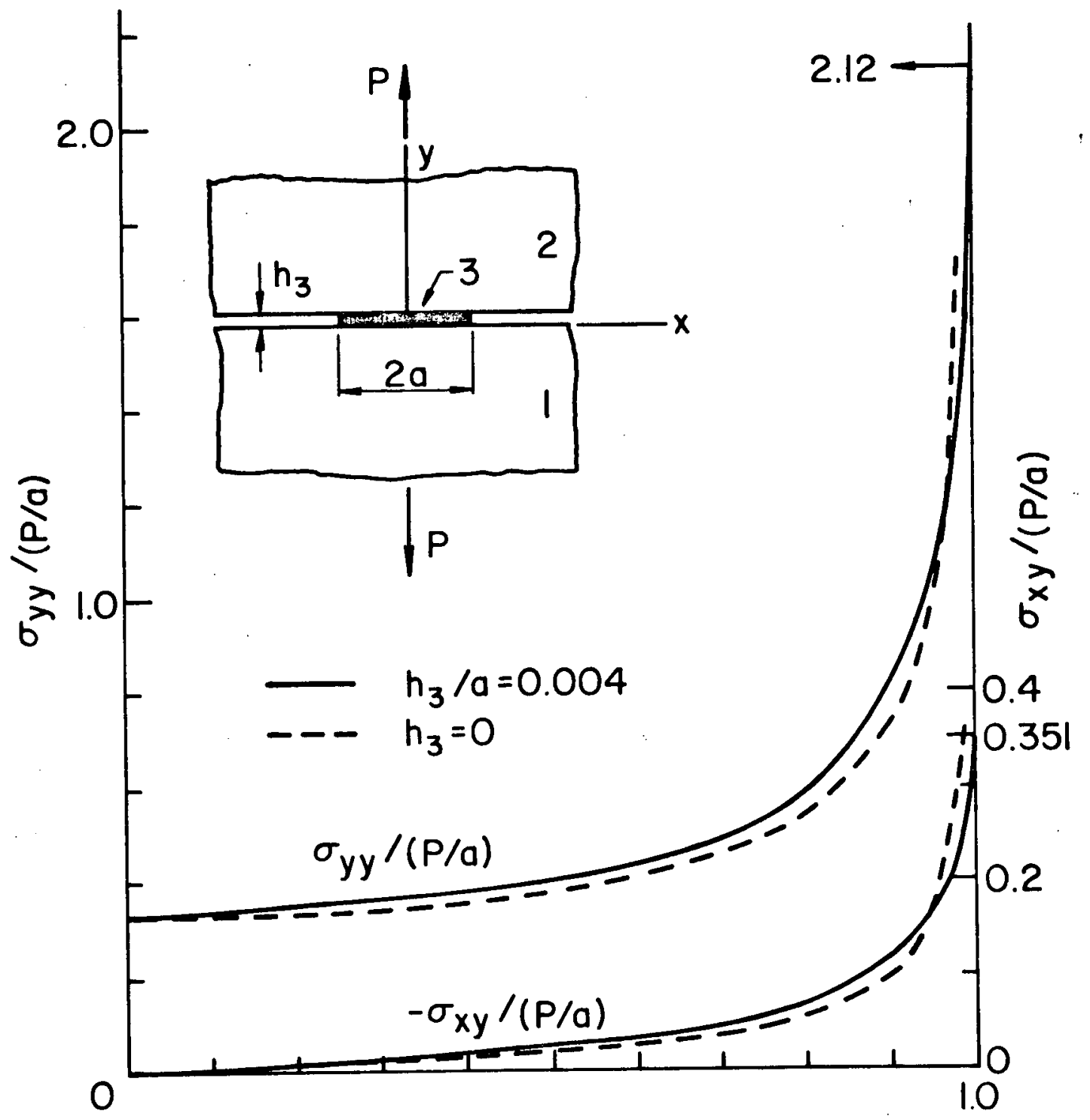

Fig. 10. Contact stresses for two elastic half planes bonded. through a thin adhesive layer. 


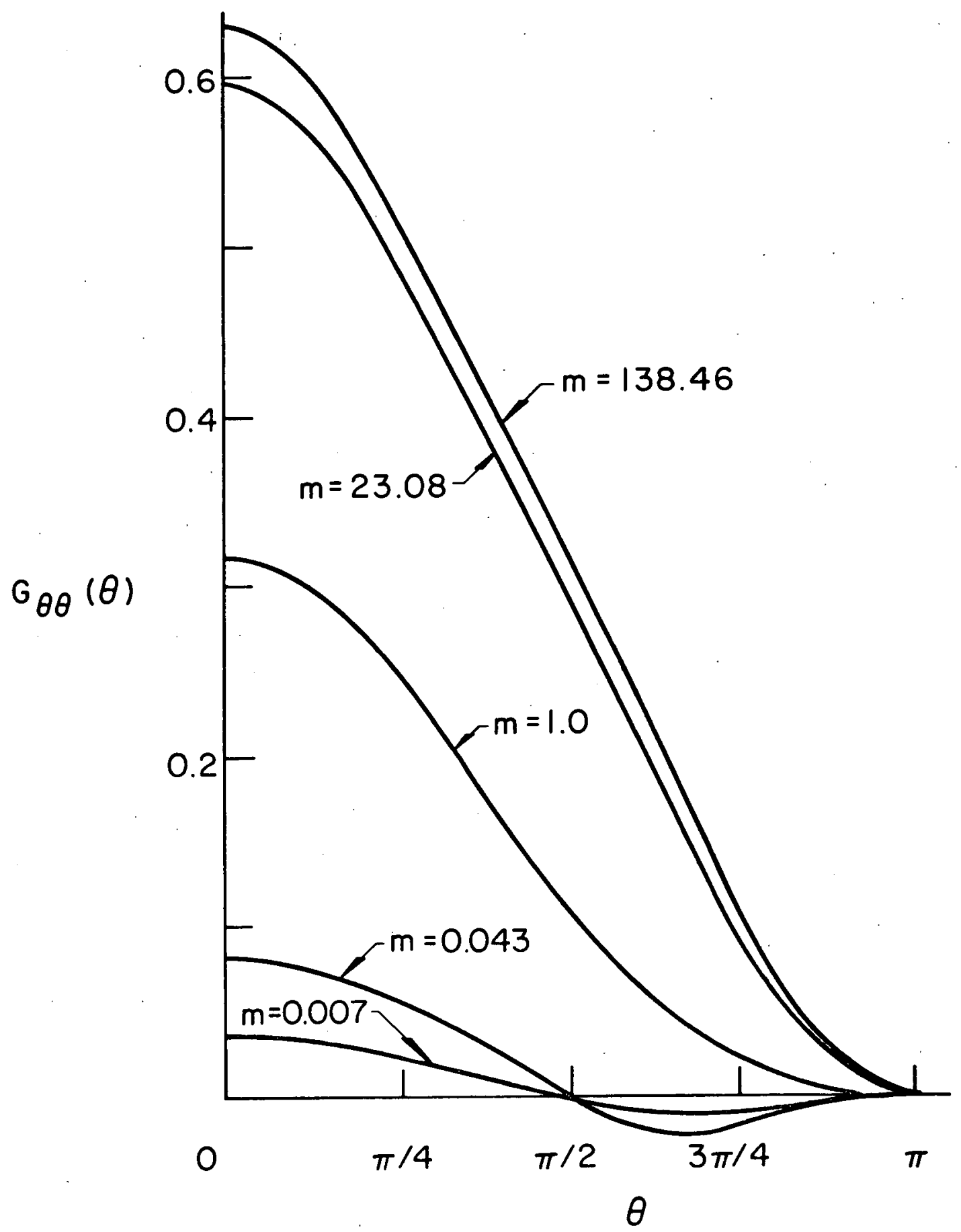

Fig. 11. Angular variation of $\sigma_{\theta \theta}$ around the crack tip terminating at an interface. 


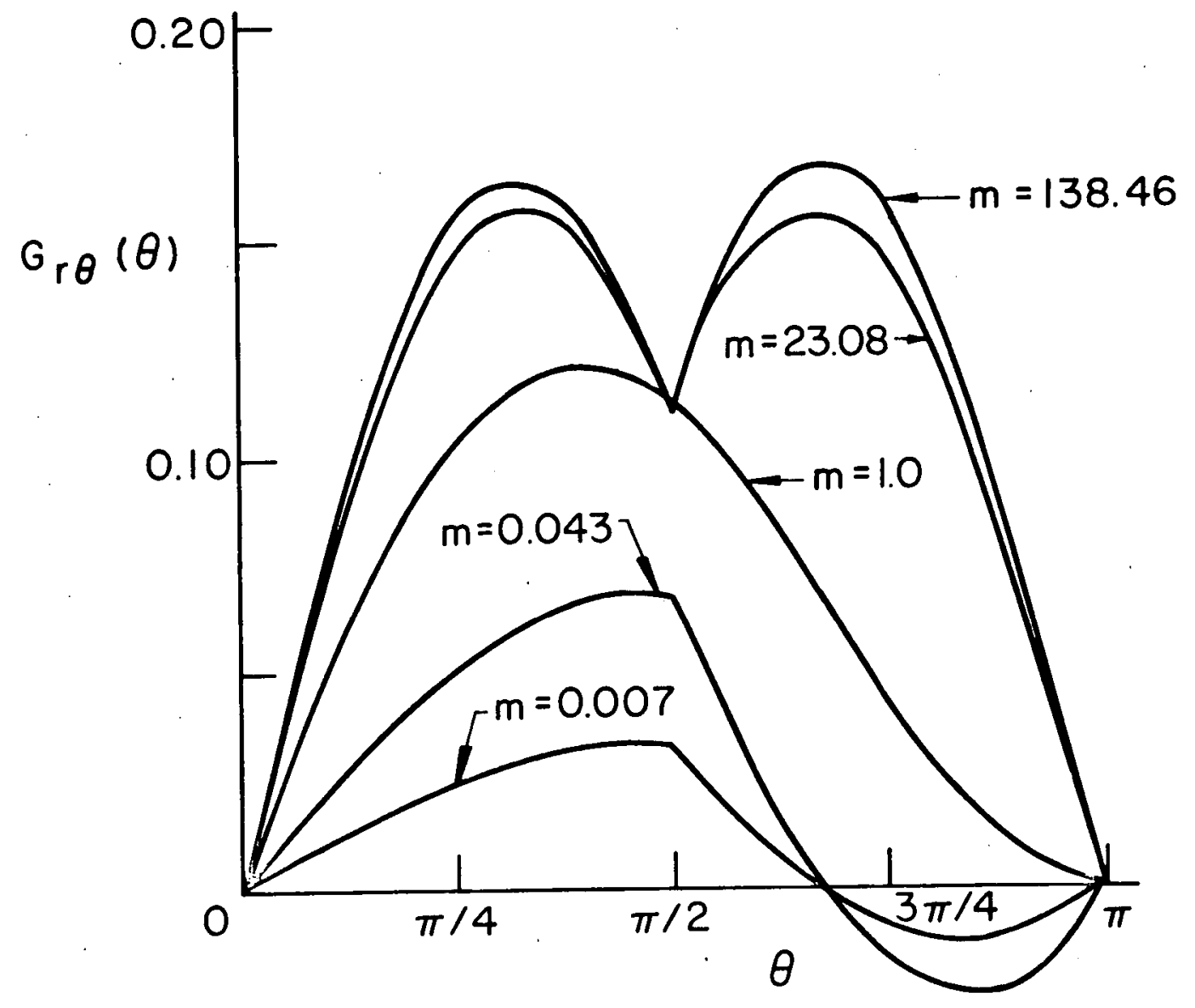

Fig. 12. Angular variation of $\sigma_{r \theta}$ around the crack tip terminating at an interface. 


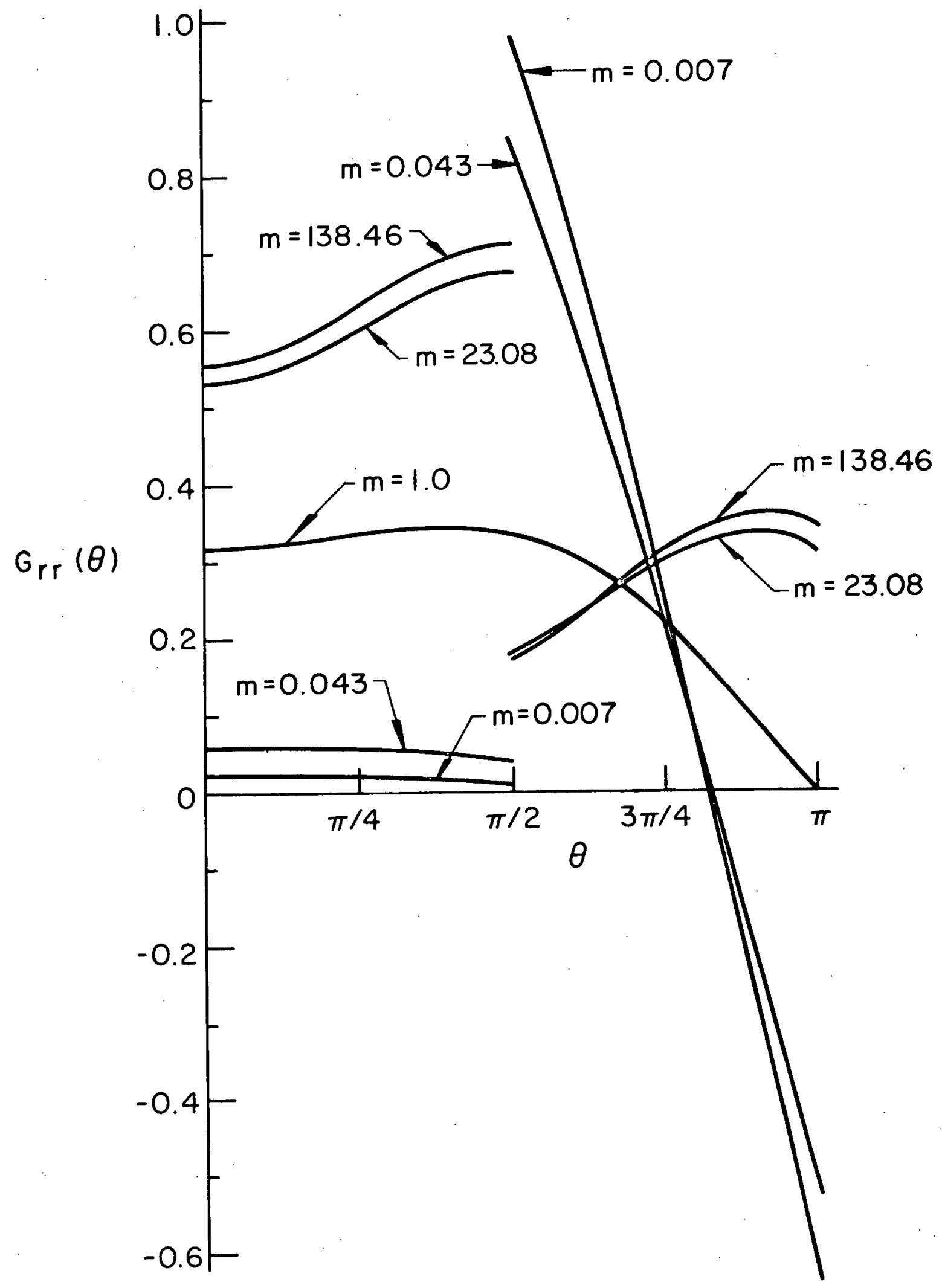

Fig. 13. Angular variation of orraround the crack tip terminating at an interface. 


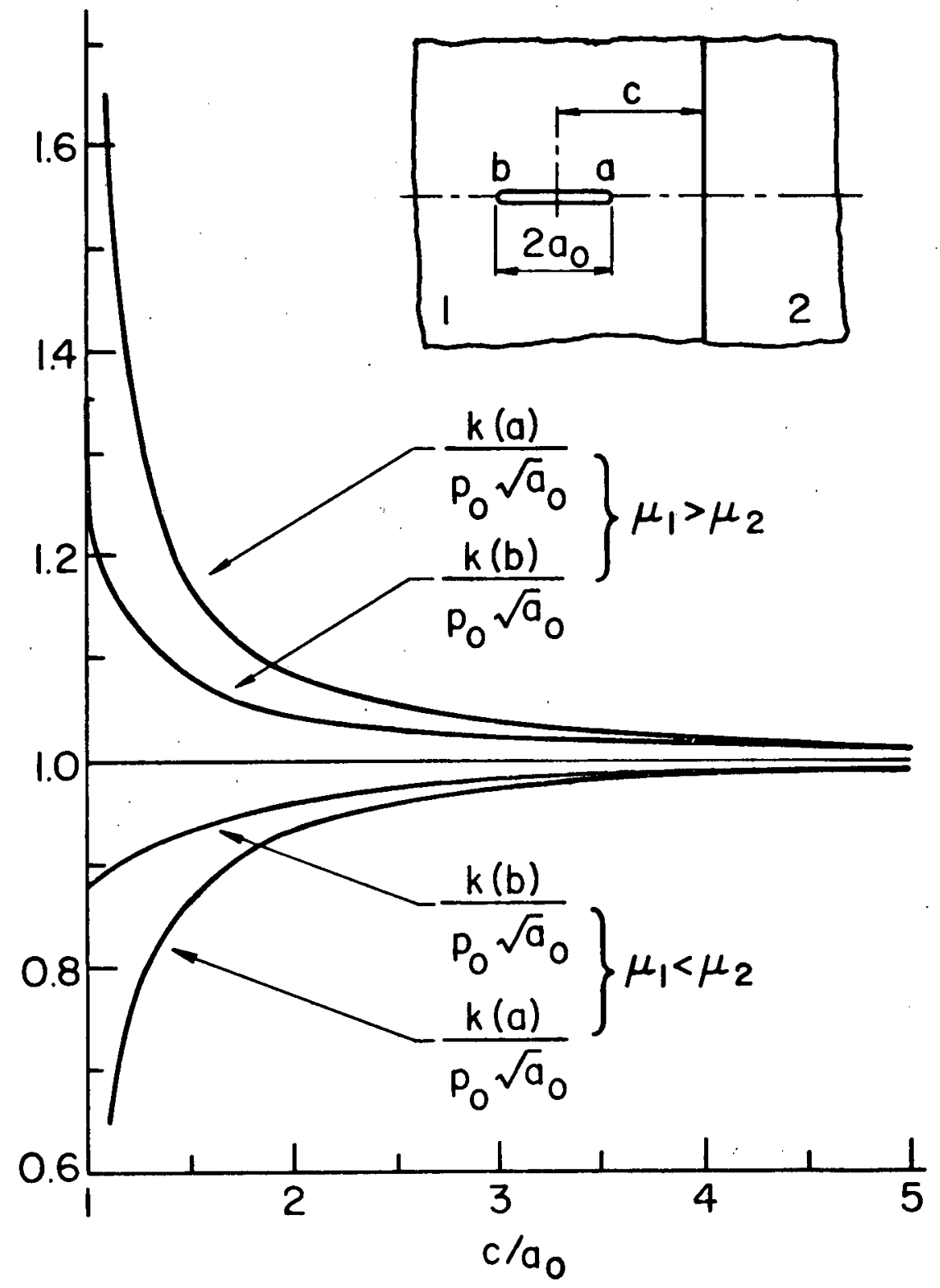

Fig. 14. Stress intensity factors for a crack perpendicular to an interface. (Materials: Aluminum and Epoxy; 1oad: crack surface pressure, $p_{0}$.) 


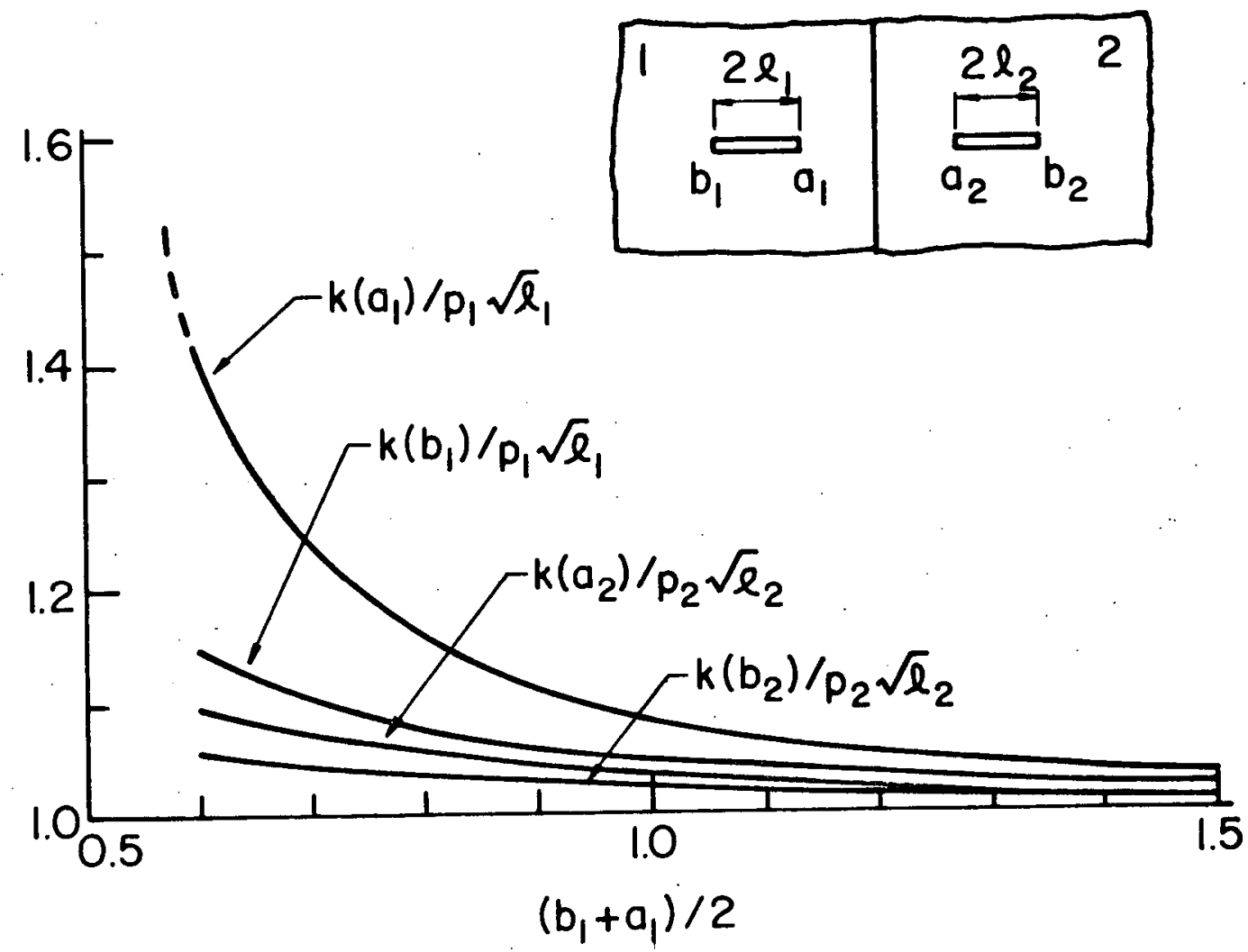

Fig. 15. Stress intensity factors for two collinear cracks imbedded in the bonded adjacent materials. (Materials: 1: A 1 uminum, 2: Epoxy; $\mathrm{a}_{2}=1=2 l_{2}=2 l_{1}, \mathrm{~b}_{2}=2 \mathrm{a}_{2}$; load: crack surface pressures $p_{1}$ and $p_{2}:\left(p_{1} / p_{2}\right)=$ $\left(E_{1} / E_{2}\right)$.) 


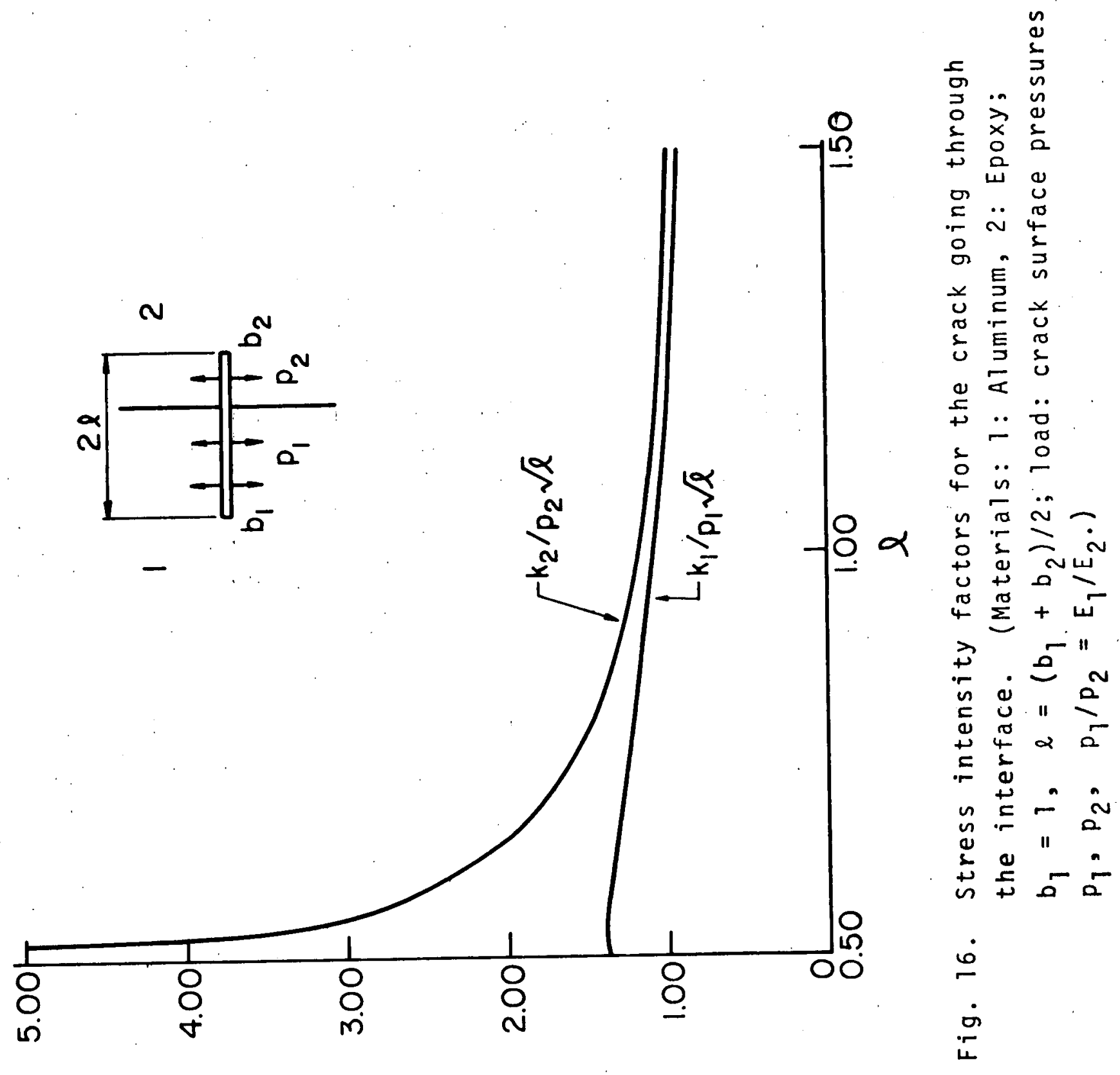




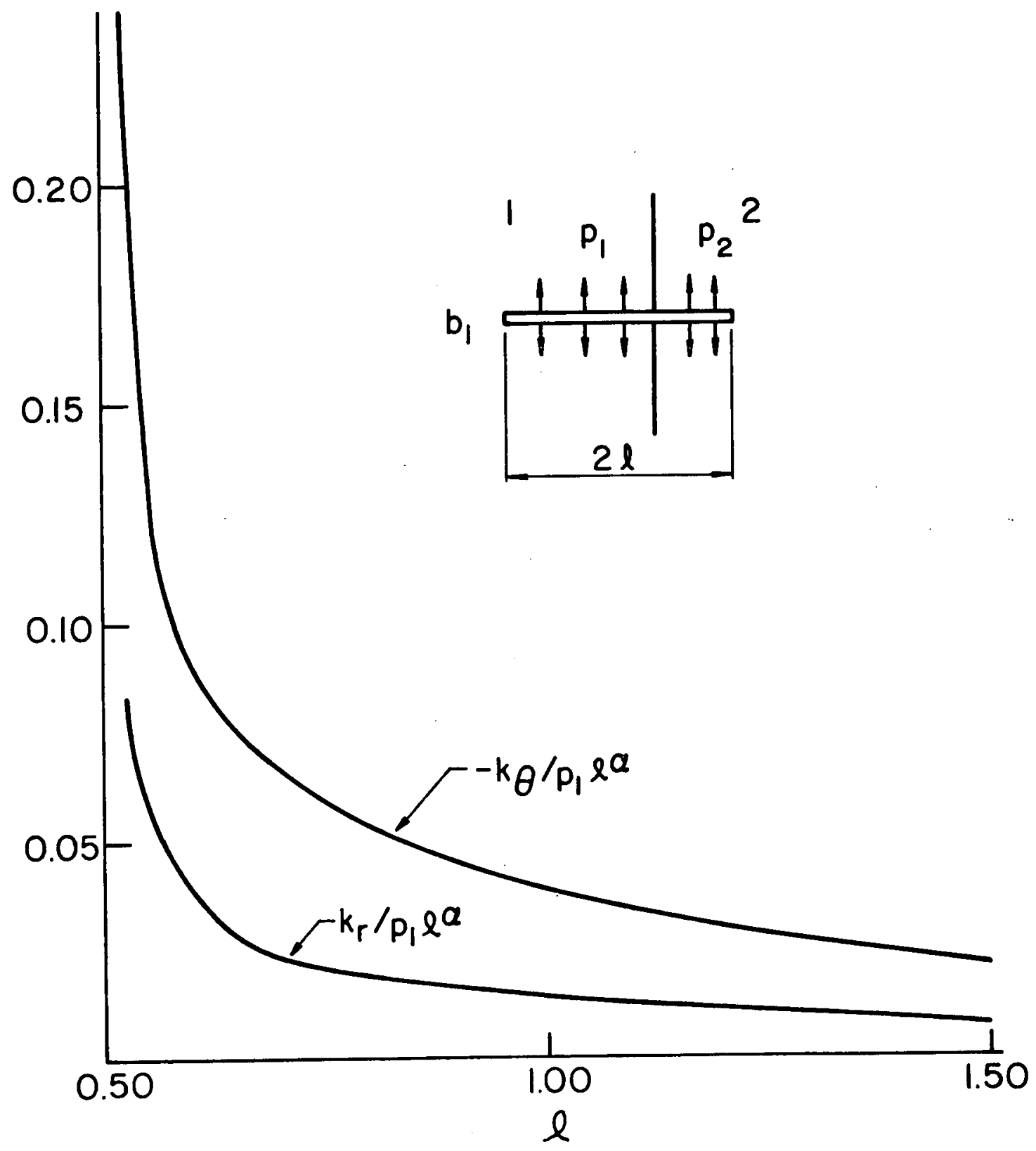

Fig. 17. Stress intensity factors for cleavage and shear stresses on the interface for a crack going through the interface. (Materials: 1: Aluminum, 2: Epoxy; $b_{1}=1$, $\ell=\left(b_{1}+b_{2}\right) / 2 ; 10 a d: c r a c k$ surface pressures $\left.p_{1}, p_{2} \cdot\right)$ 


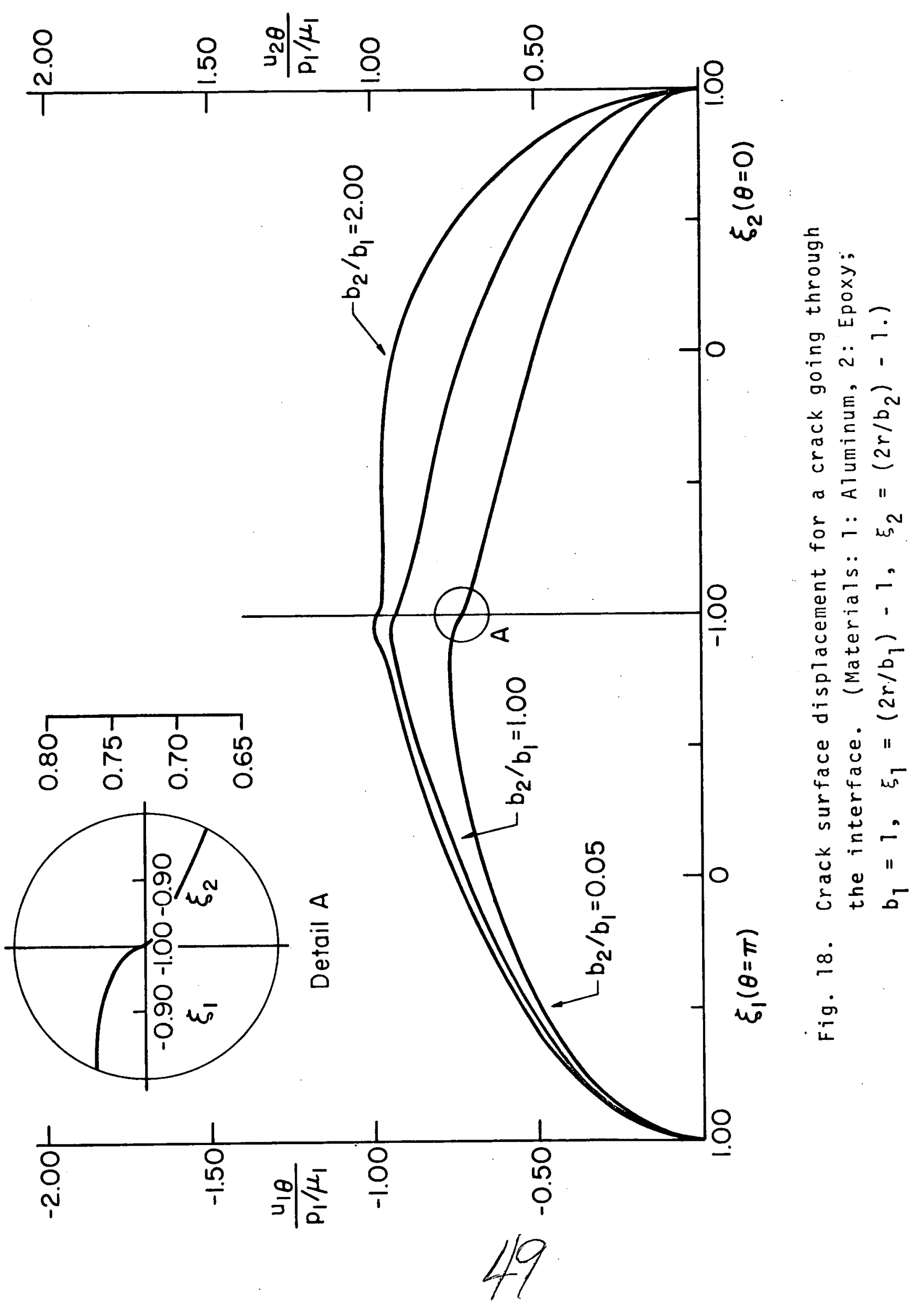




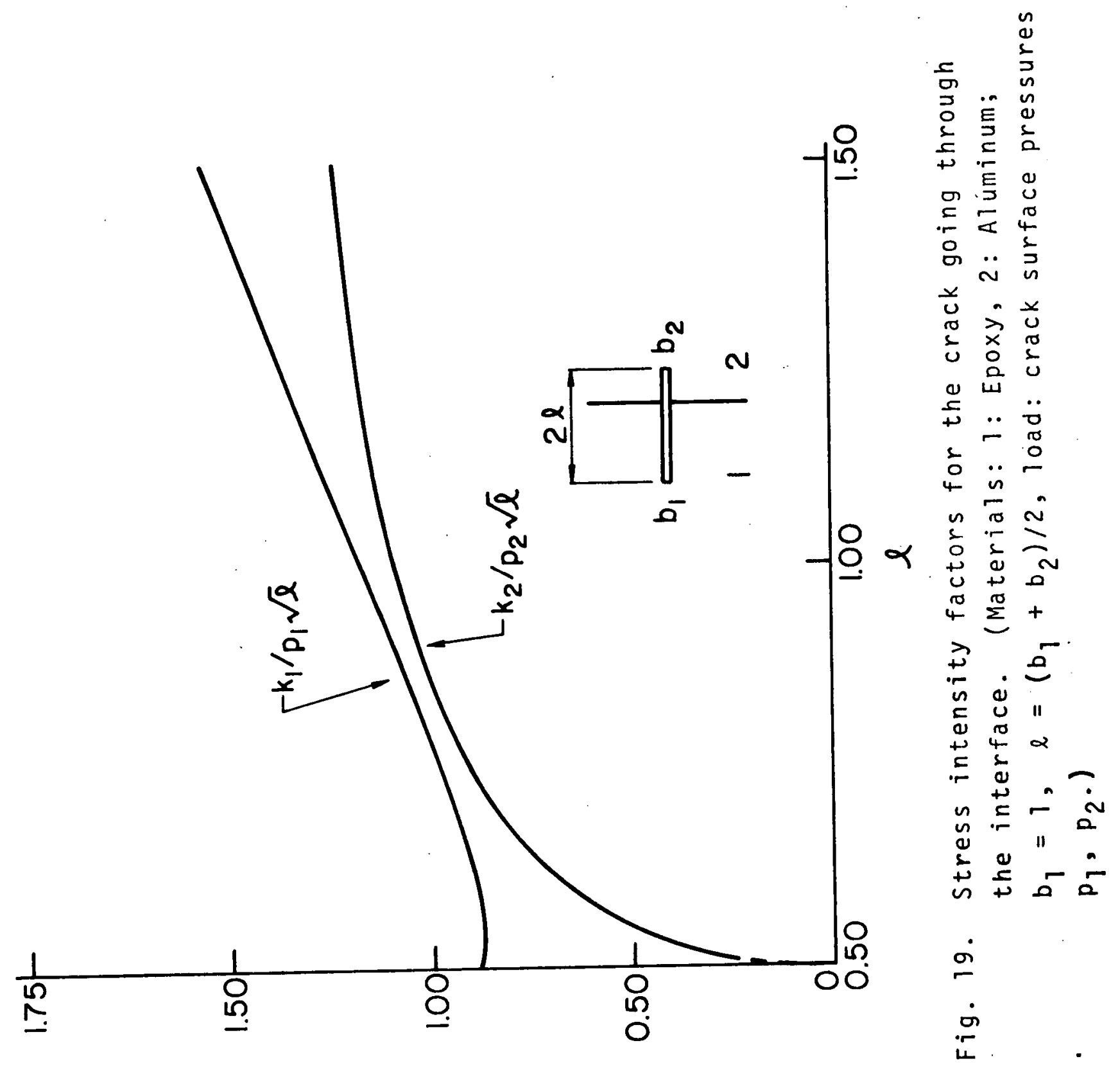

50 


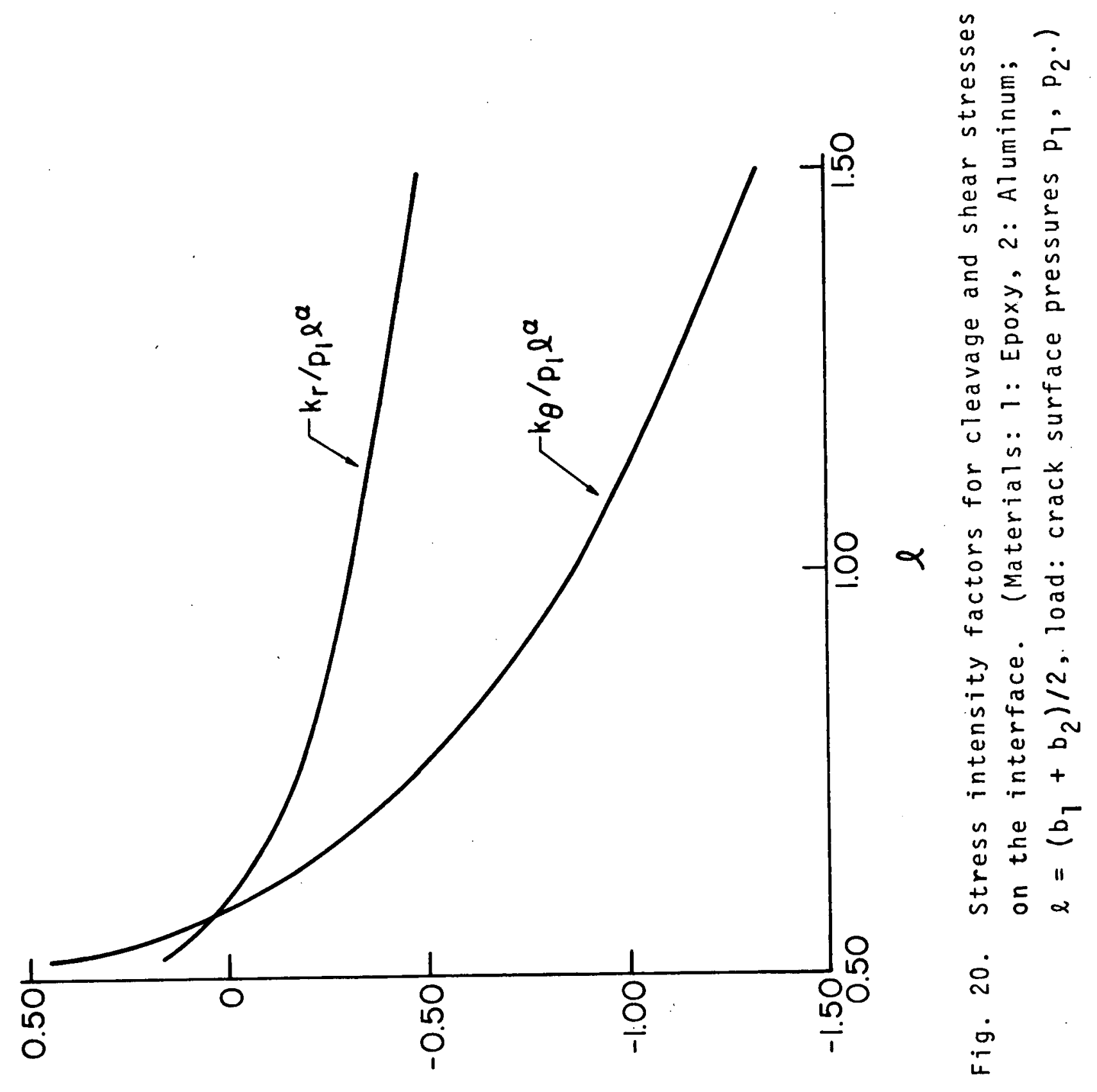




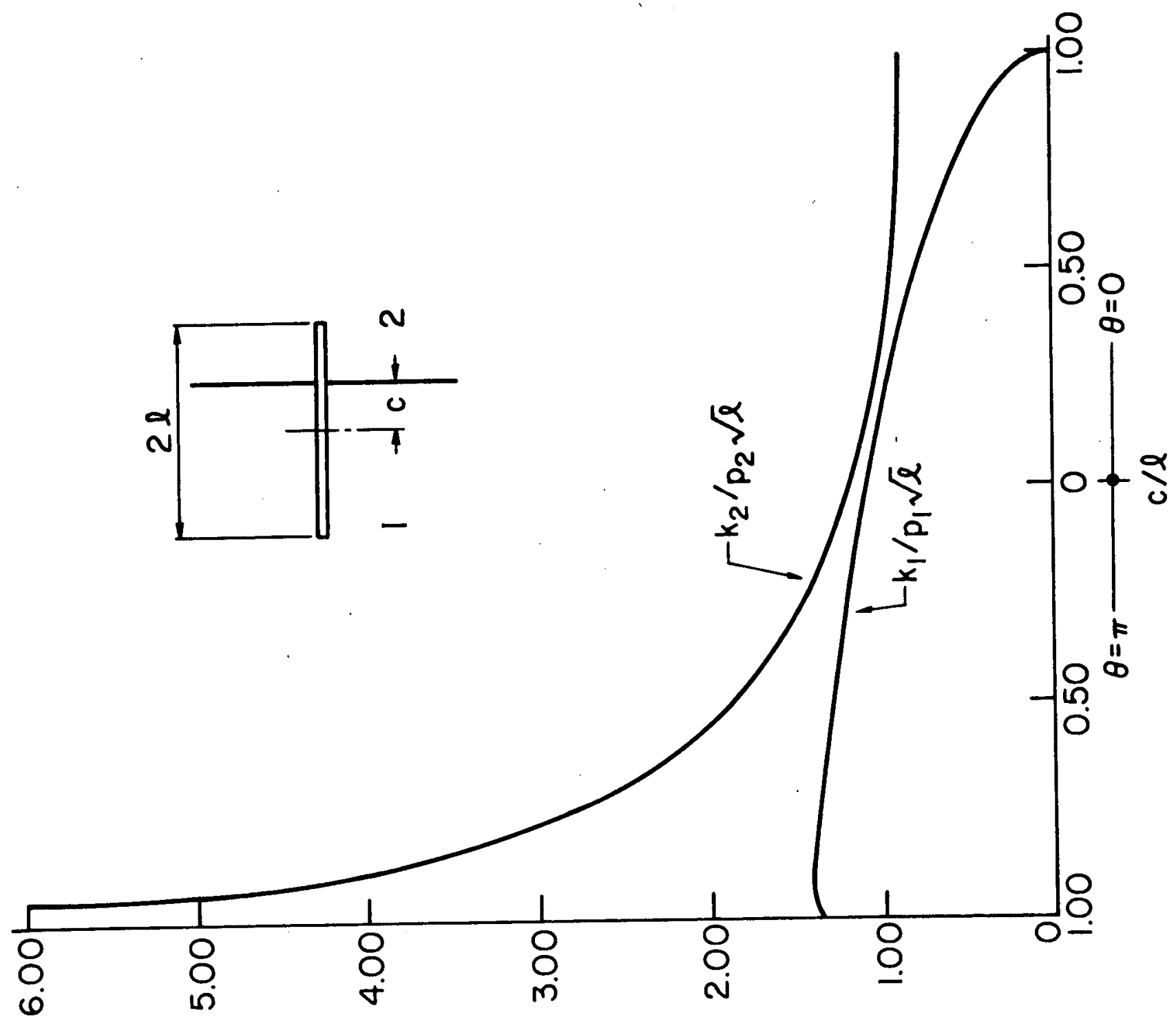

Fig. 21. Stress intensity factors for a crack going through the interface. (Materials: 1: Aluminum, 2: Epoxy; $2 l=b_{1}+b_{2}=$ constant, $c=\left|b_{1}-b_{2}\right|$; 1oad: crack surface pressures.) 


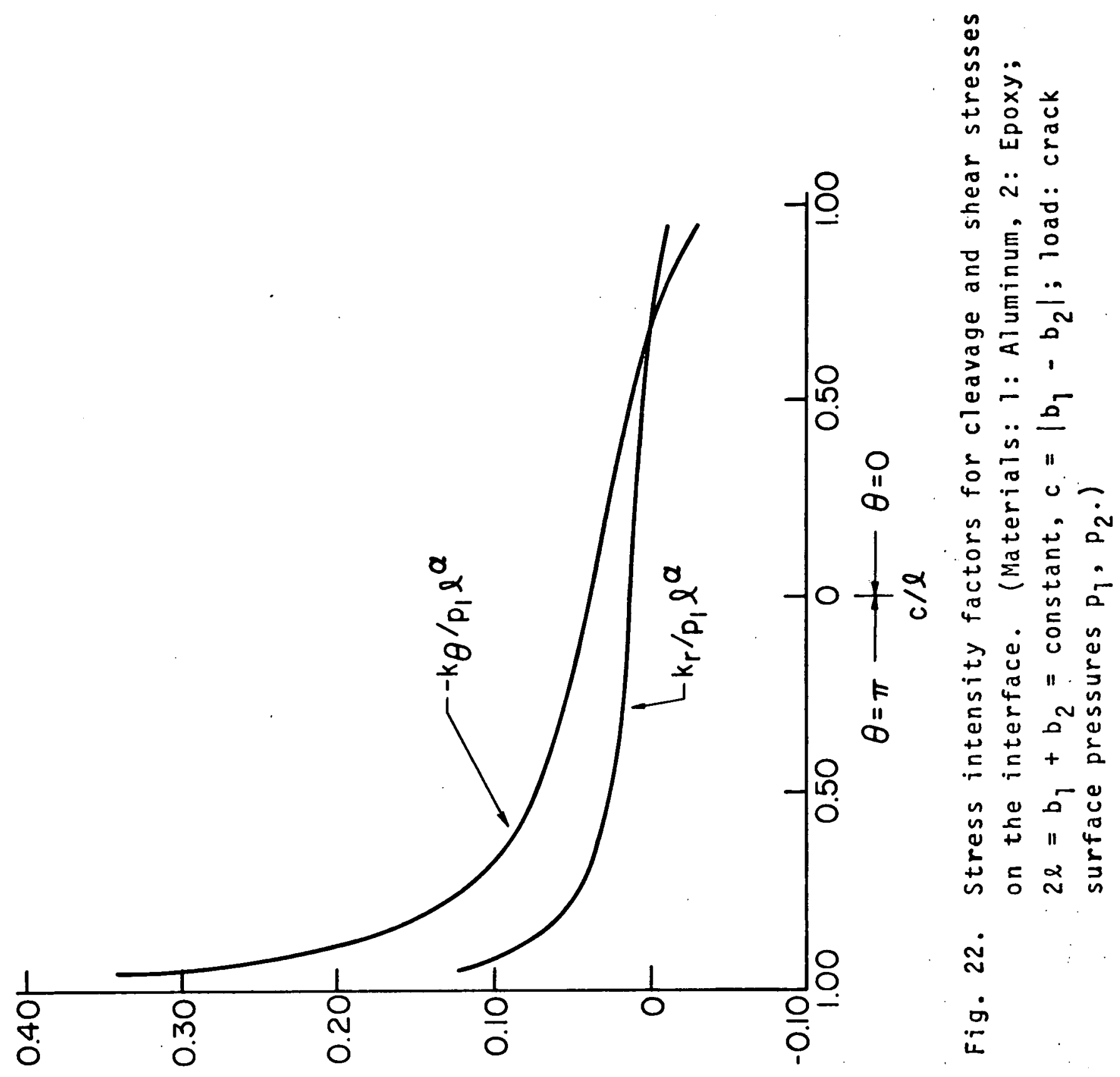




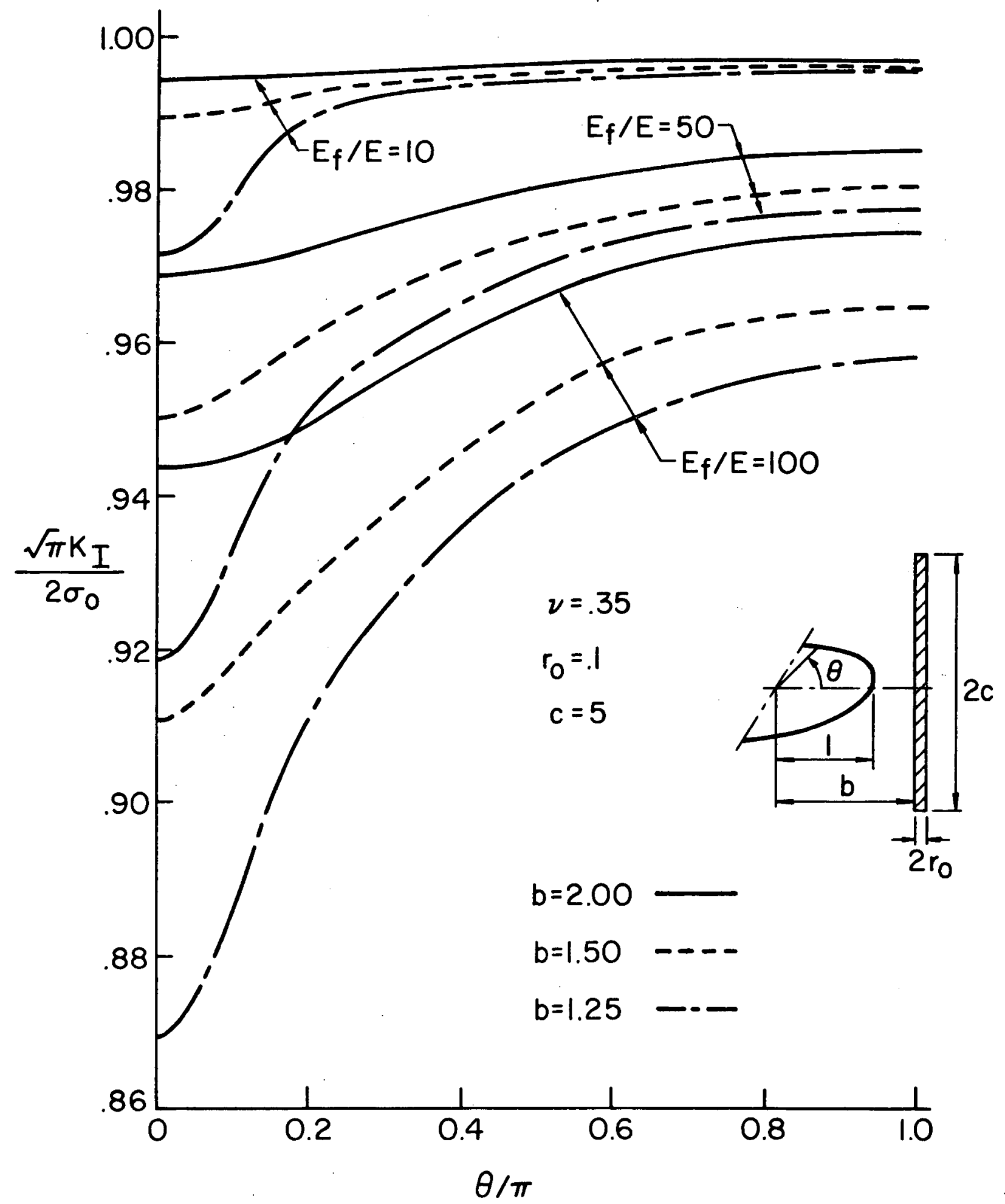

Fig. 23. Stress intensity factor on the border of a penny-shaped crack in a matrix reinforced by a single filament. ( $E_{f} / E$ : filament-to-matrix modulus ratio, b: filament distance to the crack center, crack radius = unity, $2 c$ : filament length, $r_{0}$ : filament radius, $\theta$ : angle measured from $r$ axis intersecting the filament; load: uniaxial stress $\sigma_{n}$ at infinity.) 


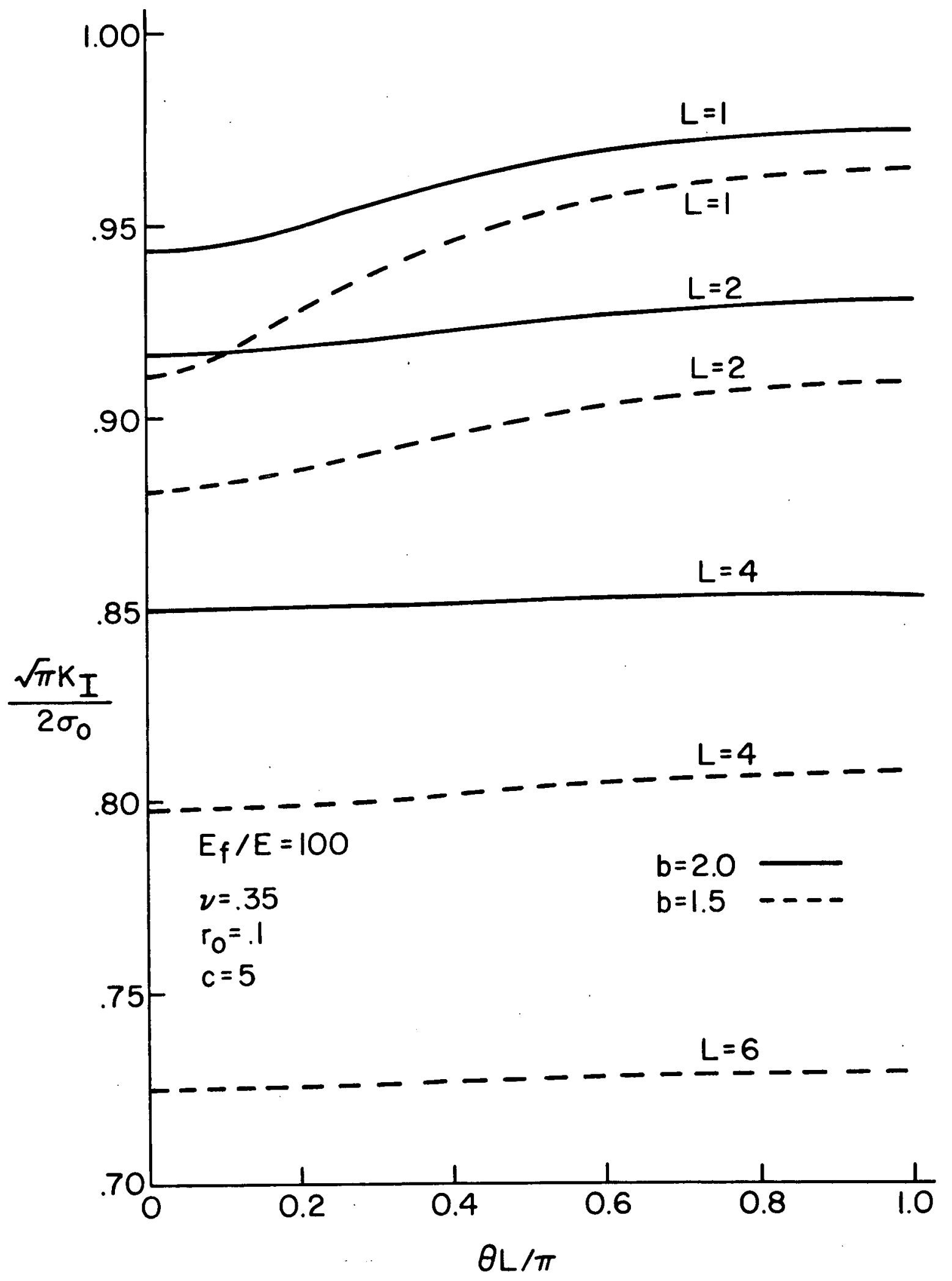

Fig. 24. The effect of filament distance $b$, and the number of filaments $L$ on the stress intensity factor for a pennyshaped crack in a filament-reinforced matrix. 


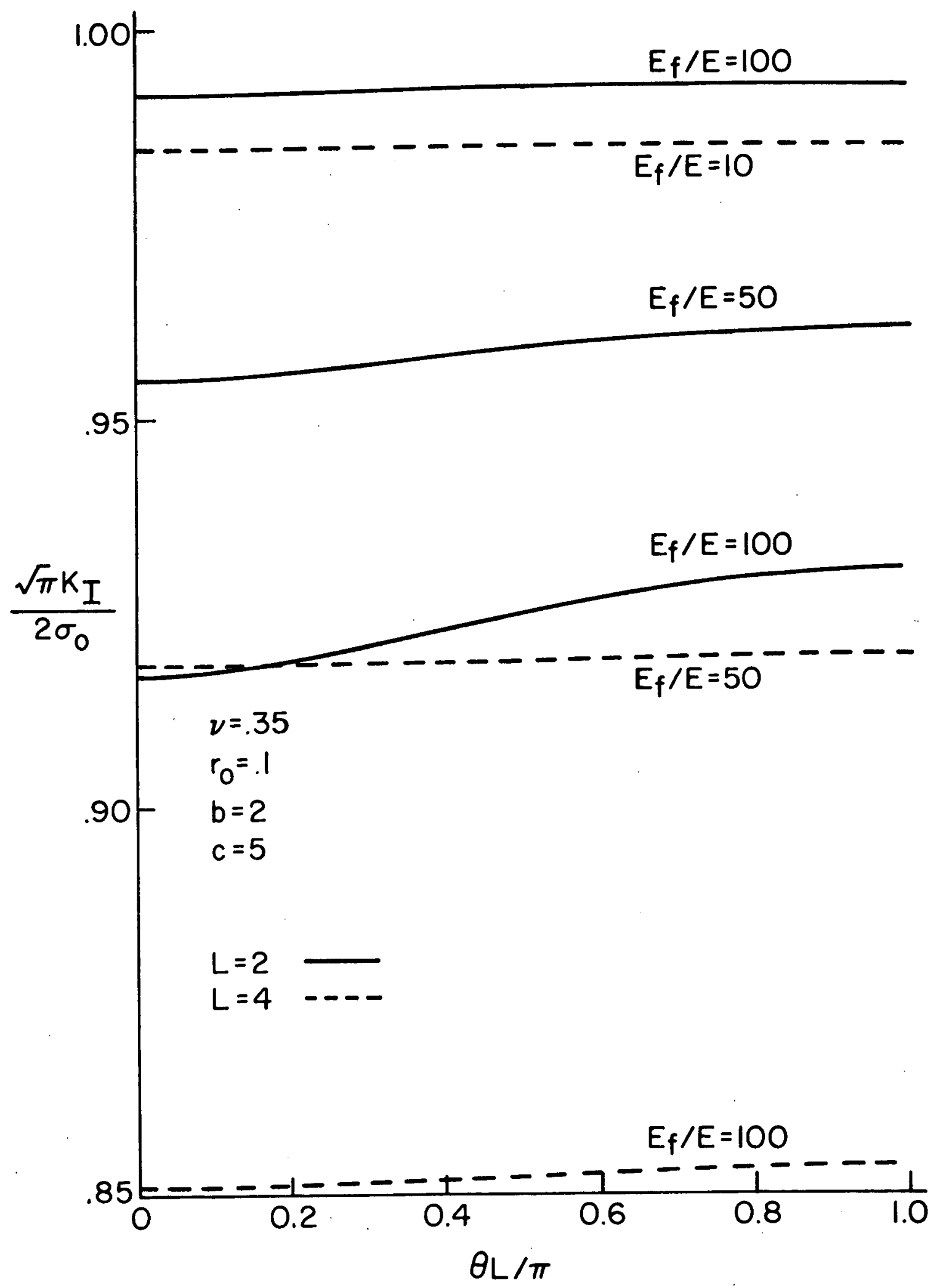

Fig. 25. The effect of modulus ratio $E_{f} / E$ on the stress intensity factor for a penny-shaped crack in a filament-reinforced matrix. 


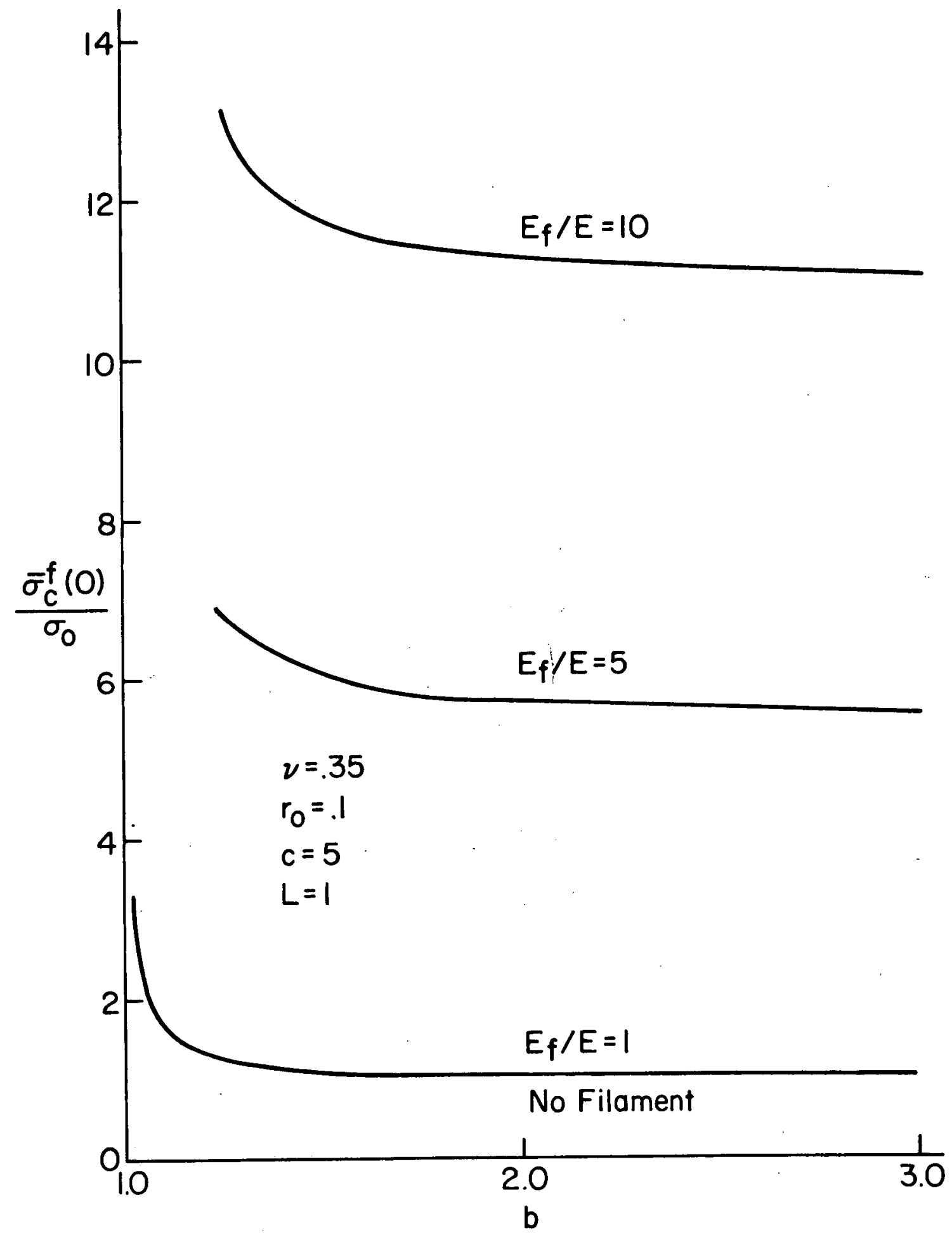

Fig. 26. The stress in the mid-plane of the filament as a function of filament distance b. (Radius of the pennyshaped crack in the matrix is the length unit.) 

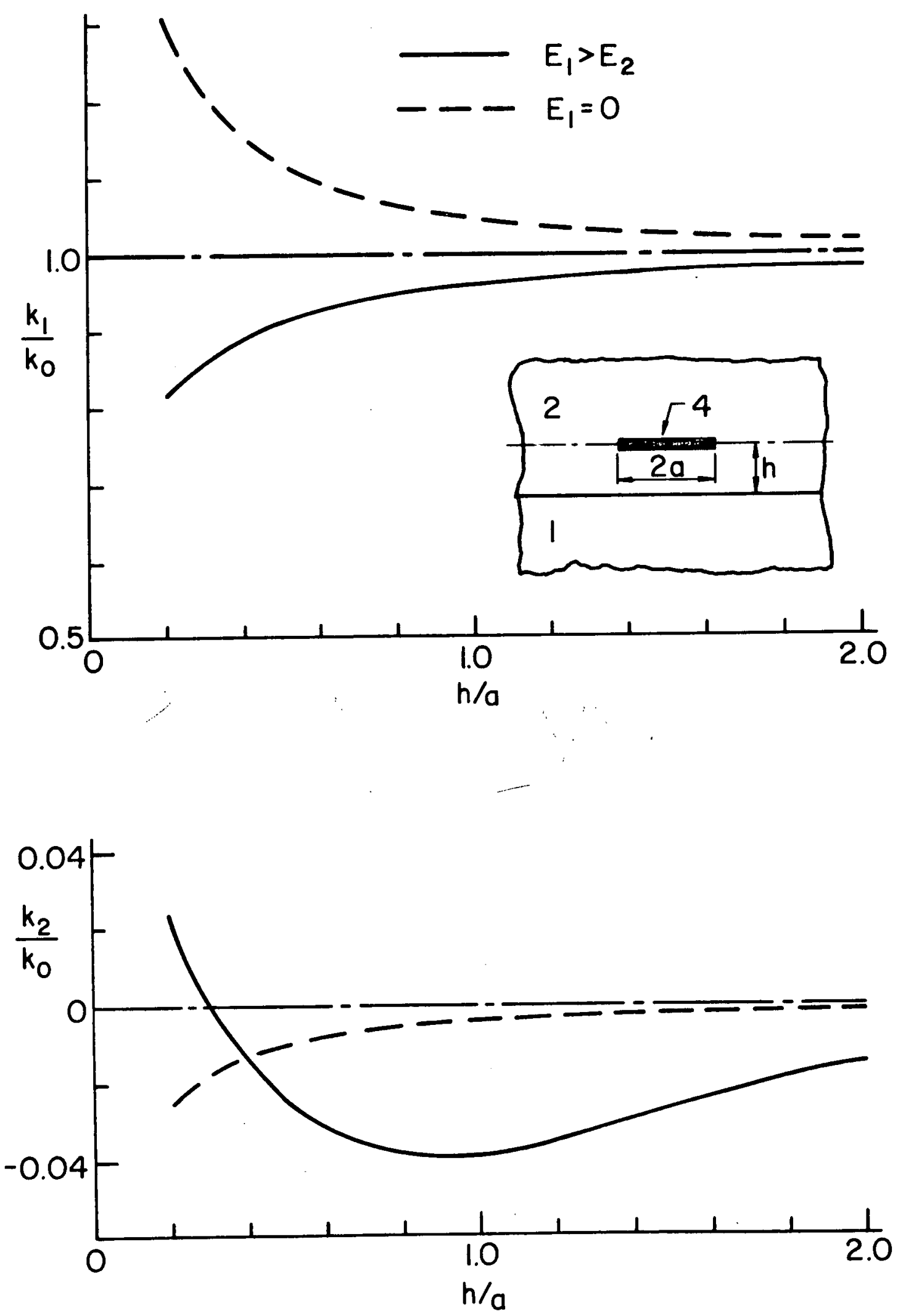

Fig. 27. Stress intensity factors for a flat rigid inclusion parallel to the bi-material interface. (Materials:

1: Aluminum, 2: Epoxy; load: displacement in the inclusion-free medium 2, $u_{0}=-\varepsilon_{0} x, v_{0}=0$; $\left.k_{0}=\mu_{2} \varepsilon_{0} a^{1 / 2}\left(k_{2}-1\right) / k_{2} \cdot\right) \quad 58$ 


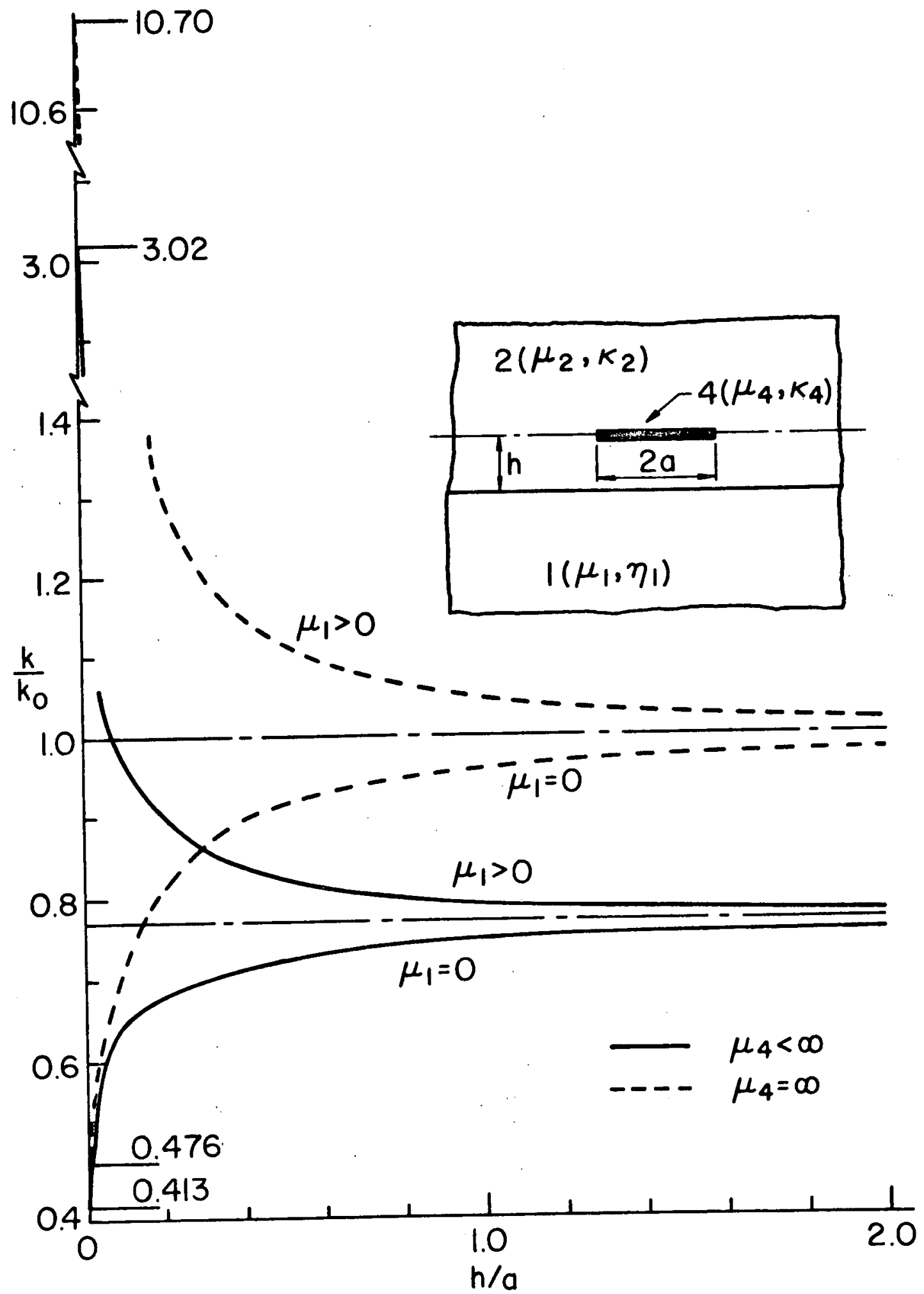

Fig. 28. Stress intensity factors for a flat elastic and for a flat inextensible inclusion lying parallel to the interface. (Materials: 1: Aluminum, 2: Epoxy, load: $\left.u_{0}=-\varepsilon_{0} x, \quad v_{0}=0 ; \quad k_{0}=\mu_{2}^{\mu^{2} \varepsilon^{a}}{ }^{1 / 2}\left(k_{2}-1\right) / k_{2} \cdot\right)$ 


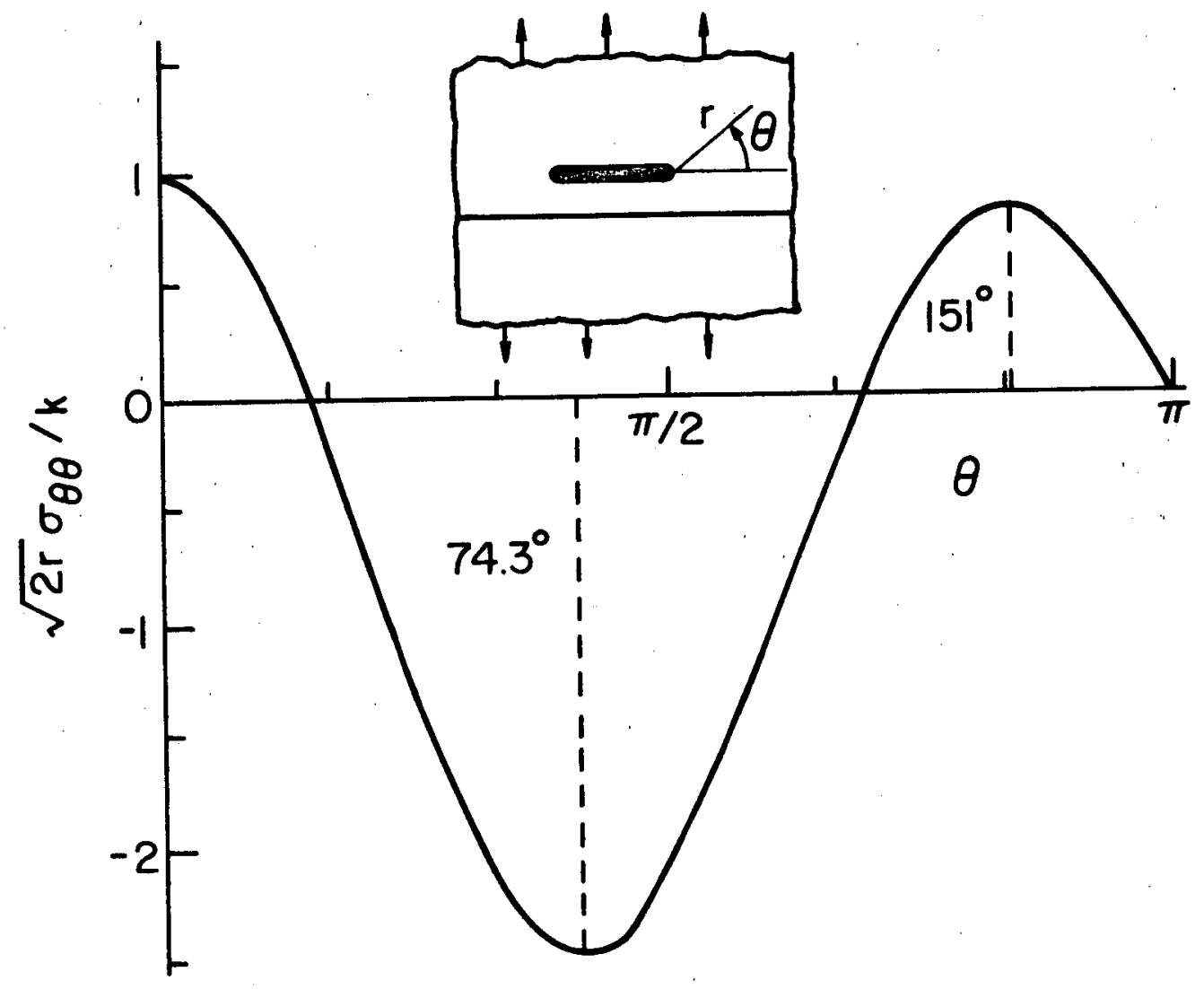

Fig. 29. $\theta$-variation of the cleavage stress around the end point of a flat elastic inclusion. 